\title{
Sensitivity analysis of hyperbolic optimal control problems
}

\author{
Adam Kowalewski • Irena Lasiecka • \\ Jan Sokołowski
}

Received: 10 March 2010 / Published online: 20 November 2010

(C) The Author(s) 2010. This article is published with open access at Springerlink.com

\begin{abstract}
The aim of this paper is to perform sensitivity analysis of optimal control problems defined for the wave equation. The small parameter describes the size of an imperfection in the form of a small hole or cavity in the geometrical domain of integration. The initial state equation in the singularly perturbed domain is replaced by the equation in a smooth domain. The imperfection is replaced by its approximation defined by a suitable Steklov's type differential operator. For approximate optimal control problems the well-posedness is shown. One term asymptotics of optimal control are derived and justified for the approximate model. The key role in the arguments is played by the so called "hidden regularity" of boundary traces generated by hyperbolic solutions.
\end{abstract}

Keywords Sensitivity analysis · Optimal control problems · Hyperbolic boundary value problems · Linear partial differential operators $\cdot$ Steklov-Poincaré operator . Kondratiev weighted spaces

\footnotetext{
A. Kowalewski

Institute of Automatics, AGH University of Science and Technology, Al. Mickiewicza 30, 30-059 Cracow, Poland

e-mail: ako@ia.agh.edu.pl

I. Lasiecka

Department of Mathematics, University of Virginia, Kerchof Hall, P.O. Box 400137, Charlottesville, VA 22904-4137, USA

e-mail: i12v@virginia.edu

J. Sokołowski ( $\varangle)$

Institut Élie Cartan, UMR 7502 Nancy-Université-CNRS-INRIA, Laboratoire de Mathématiques, Université Henri Poincaré Nancy 1, B.P. 239, 54506 Vandoeuvre Lès Nancy Cedex, France e-mail: Jan.Sokolowski@iecn.u-nancy.fr

I. Lasiecka $\cdot$ J. Sokołowski

Systems Research Institute of the Polish Academy of Sciences, ul. Newelska 6, 01-447 Warsaw, Poland
} 


\section{Introduction}

\subsection{Modelling of imperfections}

If a defect is included in the domain of integration of elliptic PDE, for example a crack, the domain becomes nonsmooth, i.e., looses the property of being Lipschitz. In such case the theory of boundary value problems defined on nonsmooth domains should be applied in order to show that the boundary value problem under consideration is well-posed in the scale of Kondratiev weighted spaces. If the size of the defect can be considered as a small parameter, the analysis can be performed on a suitable smooth domain, but the asymptotics [26-36] are derived according to the rules for singularly perturbed geometrical domains. The other possibility is to use the regular perturbations in line with homogenization techniques in optimal design: the real material is the strong material, but instead of the holes the weak material is introduced. The contrast parameter which stands for the properties of the weak material can be considered as a tool to obtain the holes by a limit passage, if necessary. This approach is useful for stationary problems, however it fails for evolution problems. This issue is particularly pronounced in low regularity hyperbolic models such as wave equations. The reason is simple, the asymptotic analysis performed for the stationary problems [42-47] gives useful information for low frequencies only, one can see this phenomenon when dealing with the spectral problems.

On the other hand, the models which are useful for applications should be simple and easy for computations. Therefore, we propose in this paper to conduct the analysis of the influence of imperfections for a simple model, just by taking only one term asymptotics of the energy functional obtained for a singular domain perturbations with nucleation of a hole. The question is whether even in such a case the presence of imperfections described in an approximate fashion destabilizes the control problems? We will show that the answer is quite complicated and a suitable regularization of the model is needed. The latter involves insertion of an additional small parameter in the boundary conditions. This parameter will force Lopatinski condition to hold for a Neumann problem which then will result in the so called "hidden regularity" [16] on the boundary. The idea of "hidden regularity" regularization has been used in the past successfully for boundary control problems - particularly in the context of numerical approximations $[5,6,13,20]$. Regularizing parameter allows to obtain smooth on the boundary approximations, which can be then taken to appropriate limits. We refer to $[1-4,8,9,17,22-25,37,39-41,48]$ for the related results on modeling and optimization of distributed parameter systems.

\subsection{Optimal control problems for the wave equation}

We consider an optimal control problem for the wave equation. The control in $L_{2}(\Gamma \times(0, T))$ is applied on a portion of lateral boundary of the cylinder $\Omega_{\rho} \times(0, T)$. We assume that in the domain $\Omega_{\rho}$ a small defect is present, in the form of a void, its size is measured by small parameter $\rho \rightarrow 0$. We want to find how the defect influences the optimal control. It seems that such analysis for the class of problems can be important for applications, with respect, for instance, to nucleation of small cracks. The exact analysis of the asymptotic behavior of optimal controls with respect to 
$\rho \rightarrow 0$ is out of reach for engineers and also quite involved. In particular, the required asymptotic analysis of hyperbolic problems for high frequencies in singularly perturbed domains is a mathematical topic still in its early stages of development. Therefore, we perform only some approximate sensitivity analysis based on asymptotic analysis of elliptic operators with respect to the parameter $\rho$. Unfortunately, such an analysis is far from being precise, more precise analysis would be based on the so-called self-adjoint extensions of elliptic operators [36], which is the topic of further studies (cf. [11]). However, even in the case of simplified analysis, the result seems to be useful and simple since we can replace the singular domain perturbations in $\Omega_{\rho}$ by the regular perturbations in boundary conditions on the truncated domain $\Omega_{R}$. Here, we use the idea which can be very useful in the domain decomposition technique for the numerical solution of hyperbolic equations. We have also a precise result of sensitivity analysis, interesting on its own i.e., one term asymptotic expansion of the optimal control is obtained with respect to the parameter $\rho$ for the control problem with constraints. The solutions considered are of "finite energy" controlled by physically significant $L_{2}(\Sigma)$ boundary inputs.

From the PDE point of view, the main difficulty of the problem is due to intrinsic low regularity of solutions to hyperbolic problems driven by $L_{2}$ Neumann boundary data. Of particular relevance is the regularity due to non-homogeneous boundary data which undergo infinitesimal perturbations. Standard hyperbolic regularity, is of no use in such analysis. What is essential instead, is the so-called "hidden regularity" property displayed by hyperbolic flows which satisfy the Lopatinski condition $[7,16,18,19,38]$. However, the model under consideration is equipped with the Neumann type of boundary conditions where hidden regularity does not hold [18, 19] unless the dimension of the domain is equal to one. Thus, the additional technical difficulty is related to the Neumann control in $L_{2}(\Gamma \times(0, T))$ where Lopatinski condition fails. This has implications on regularity theory which leads to the loss of $1 / 3$ derivative when analyzing the control-input map [18]. In order to deal with this difficulty, we shall impose absorbing boundary conditions (typical boundary friction) which can also be considered as a feedback stabilizer for the wave equation $[12,13$, 20]. These boundary conditions, while producing long time stabilizing effect allow also to prove a weak version of "hidden regularity" for finite energy solutions [5, 6, $13,16]$. This latter property turns out critical for the analysis of sensitivity conducted in the present work. We shall show that the resulting control problem is well-posed, with the unique optimal control, and the first order perturbation of the optimal control with respect to the parameter $\rho$ is uniquely determined by the solution of the control problem in the unperturbed domain. In other words, for a small defect in the domain of integration, its influence on the optimal control is determined by solving an auxiliary optimal control problem in unperturbed domain. Such an information could be useful for practical purposes, since the cost of numerical solution in singularly perturbed geometrical domain could be substantially higher, due to the singularities, compared to the cost of numerics performed on smooth unperturbed domains. It should be noted that the idea of "hidden regularity" regularization, in the context of wave equation, has been explored in the past. For instance, [5, 6] appplies the same regularization to approximation of Riccati operators arising in boundary control of wave equation with Neumann boundary conditions. In fact, the entire theory of con- 
Fig. 1 The domain $\Omega_{\rho}$ in two spatial dimensions

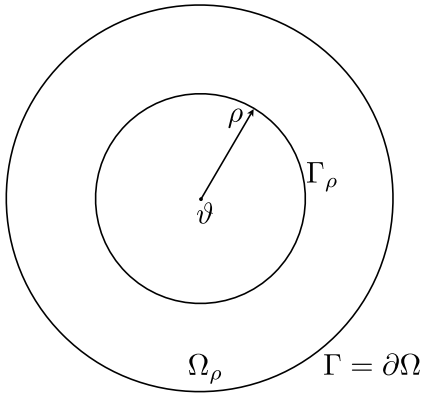

vergence of FEM approximations of Riccati solutions rests on a suitable prior "hidden" regularization of the problem. The passage with the limit on the parameter of regularization leads to the ultimate convergence result for the original wave equation. The method of "hidden" regularization has been also used in domain decomposition procedures introduced and described in [13].

\section{Geometry of $\Omega_{\rho}$}

To fix the ideas, we consider the following model problem. Let $\Omega \subset \mathbb{R}^{n}$ be a domain with smooth boundary $\Gamma$ and $\mathbb{B}_{\rho}$ be a defect included in $\Omega$, in the form of a void. The case of a small crack can also be considered in our framework. The domain with the defect is denoted by $\Omega_{\rho}$ (Fig. 1). Usually, if the asymptotic analysis in singularly perturbed domains is applied for the construction of an approximate problem of simpler nature, some attributes of the defect like [26, 37] mass matrix, polarization matrix etc. are necessary in order to replace the domain $\Omega_{\rho}$, which is singularly perturbed, by a punctured domain which remembers the presence of the defect by means of a singular potential located at the center of the defect. Here we avoid this type of approximation, we apply only the non-local Steklov-Poincaré operator which results from the asymptotic energy expansion for the Laplacian. This operator depends on the small parameter $\rho \rightarrow 0$, and we can use its expansion with respect to $\rho$ in order to obtain the constructive formulae from our sensitivity analysis. For simplicity we fix the spatial dimension $n=2$.

We denote by

$$
\begin{aligned}
& \Omega_{\rho}=\Omega \backslash \overline{\mathbb{B}}_{\rho} \subset \mathbb{R}^{2}, \\
& \partial \Omega_{\rho}=\Gamma \cup \Gamma_{\rho},
\end{aligned}
$$

where: $\Omega$ is a domain on the plane $\mathbb{R}^{2}$ with a smooth boundary $\partial \Omega$ and

$$
\mathbb{B}_{\rho}=\{x:|x-\vartheta|<\rho\}
$$

with a smooth boundary $\Gamma_{\rho}$.

\section{Domain decomposition $\Omega=\mathbb{B}_{R} \cup \Gamma_{R} \cup \Omega_{R}$}

Another useful geometrical construction for our problem is based on the domain decomposition technique. The idea is simple, we want to perform the asymptotic 
Fig. 2 The domain $\Omega_{R}$

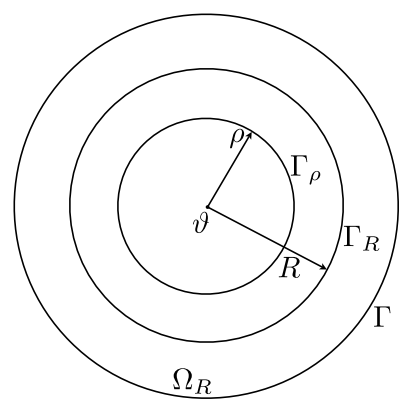

analysis with respect to singular perturbations of domains in subdomain $\mathbb{B}_{R}$ with $R>\rho$ fixed once forever. The goal is then to study the influence of the small parameter $\rho \rightarrow 0$ on the optimal control in disjoint subdomain $\Omega_{R}$. This decomposition allows us to obtain a simple problem in $\Omega_{R}$, with regular perturbations of the boundary conditions imposed on the interface $\Gamma_{R}$ between two subdomains. In this way we introduce the new hyperbolic problem to be considered, defined in the cylinder $\Omega_{R} \times(0, T)$, and avoid in fact any interaction with the boundary layer created in $\mathbb{B}_{R}$ by the presence of the defect. However, we need the preliminary analysis of the defect on the Steklov-Poincaré operator which lives on $\Gamma_{R}=\partial \mathbb{B}_{R}$. This analysis is performed only for the elliptic operator, and we use the result to construct the asymptotics for the elliptic Steklov-Poincaré operator. We consider the geometry from the figure below. Let us surround $\Gamma_{\rho}$ by the circle $\Gamma_{R}$ such that $R>\rho>0$ (Fig. 2). Consequently, we denote

$$
\Omega_{R}=\Omega \backslash \overline{\mathbb{B}}_{R}
$$

where:

$$
\mathbb{B}_{R}=\{x:|x-\vartheta|<R\}
$$

and we assume that the centre $\vartheta:=\mathcal{O}$ is just the origin.

For further purposes we set the non-local Neumann boundary condition on $\Gamma_{R}$ :

$$
-\frac{\partial y}{\partial n}=A_{\rho}(y) \quad \text { on } \Gamma_{R}
$$

where: $A_{\rho}$ is a Steklov-Poincaré operator defined below in the domain $C(R, \rho)=$ $\mathbb{B}_{R} \backslash \overline{\mathbb{B}}_{\rho}$. The operator $A_{\rho}$ is a mapping of $H^{1 / 2}\left(\Gamma_{R}\right) \mapsto H^{-1 / 2}\left(\Gamma_{R}\right)$. We recall the definition of the operator.

For given element $v \in H^{1 / 2}\left(\Gamma_{R}\right)$ we solve the boundary value problem

$$
-\Delta w=0 \quad \text { in } C(R, \rho), \quad \frac{\partial w}{\partial v}=0 \quad \text { on } \Gamma_{\rho}, \quad w=v \quad \text { on } \Gamma_{R},
$$

and set

$$
A_{\rho} v=\frac{\partial w}{\partial v} \quad \text { on } \Gamma_{R},
$$

where $v$ is the unit exterior normal vector on $\partial C(R, \rho)$, note that the unit exterior normal vector $n$ on $\Gamma_{R} \subset \partial \Omega_{R}$ is $n=-v$. 
Notation The following notation will be adopted.

We shall denote by $|u|_{s, \Omega} \equiv|u|_{H^{s}(\Omega)}$ Sobolev's norm of order $s$ defined on a domain $\Omega$. Similarly, the inner products $(u, v)_{\Omega}=\int_{\Omega} u v d x$ denote $L_{2}$ products integrated over $\Omega$. $(u, v)_{\Gamma}$ denotes $L_{2}(\Gamma)$ inner product. The same notation will be used for $\Gamma_{R}$.

Trace operators or restrictions to the boundary are denoted by $\left.\gamma w \equiv w\right|_{\Gamma}$, and $\left.\gamma_{R} w \equiv w\right|_{\Gamma_{R}}$

Time-space function spaces are denoted by $C(X) \equiv C([0, T] ; X), L_{p}(X) \equiv$ $L_{p}(0, T ; X)$ where $X$ is a given Banach space.

Projection operators $P_{i}: \mathbb{R}^{2} \mapsto \mathbb{R}^{1}$ are given by $P_{i}\left(u_{1}, u_{2}\right)=u_{i}, i=1.2$.

The Constant $C$ or $c$ denote generic constants that do not depend on solution or the parameter $\rho$.

We begin with the following preliminary Lemma which is known to the specialists.

Lemma 1 For all $\rho \geq 0$ the operator $A_{\rho}: L_{2}\left(\Gamma_{R}\right) \mapsto L_{2}\left(\Gamma_{R}\right)$ is self-adjoint and it is continuous $H^{1 / 2}\left(\Gamma_{R}\right) \mapsto H^{-1 / 2}\left(\Gamma_{R}\right)$. Moreover, for all $u \in H^{1 / 2}\left(\Gamma_{R}\right)$ the following bounds are uniform in $\rho \in[0,1]$.

$$
\begin{gathered}
\left(A_{\rho} u, u\right)_{L_{2}\left(\Gamma_{R}\right)}=\left|A_{\rho}^{1 / 2} u\right|_{\Gamma_{R}}^{2} \sim|u|_{H^{1 / 2}\left(\Gamma_{R}\right)}^{2}=|u|_{1 / 2, \Gamma_{R}}^{2}, \\
c|u|_{H^{1 / 2}\left(\Gamma_{R}\right)}^{2} \leq\left|A_{\rho}^{1 / 2} u\right|_{\Gamma_{R}}^{2} \leq C|u|_{H^{1 / 2}\left(\Gamma_{R}\right)}^{2} .
\end{gathered}
$$

Proof We denote by $D: L_{2}\left(\Gamma_{R}\right) \mapsto L_{2}\left(\Omega_{R}\right)$ the Dirichlet map defined as

$$
D v \equiv w, w \quad \text { given by (6) }
$$

By using Green's formula one finds that for all $v, z \in H^{1 / 2}\left(\Gamma_{R}\right)$ one has

$$
\begin{aligned}
\left(A_{\rho} v, z\right)_{\Omega_{R}} & =\left(\frac{\partial}{\partial v} D v, D z\right)_{\Gamma_{R}}=\left(\frac{\partial}{\partial v} D v, D z\right)_{\Gamma_{R} \cup \Gamma_{\rho}} \\
& =(\Delta D v, D v)_{\Omega_{R}}+(\nabla D v, \nabla D z)_{\Omega_{R}}=(\nabla D v, \nabla D z)_{\Omega_{R}}
\end{aligned}
$$

which shows self-adjointness. In addition

$$
\left(A_{\rho} v, z\right)_{\Omega_{R}} \leq|D v|_{1, \Omega_{R}}|D z|_{1, \Omega_{R}} \leq C|v|_{1 / 2, \Gamma_{R}}|z|_{1 / 2, \Gamma_{R}}
$$

and

$$
\left(A_{\rho} v, v\right)_{\Omega_{R}}=|\nabla D v|_{\Omega_{R}}^{2} \sim|v|_{1 / 2, \Gamma_{R}}^{2} .
$$

Since $\nabla D v \in L_{2}(\Omega)$ implies that $D v \in H^{1}(\Omega)$ and $\left.D v\right|_{\Gamma_{R}} \in H^{1 / 2}\left(\Gamma_{R}\right)$, we infer that $v \in H^{1 / 2}\left(\Gamma_{R}\right)$. The above concludes, via Closed Graph Theorem, the conclusion stated in Lemma 1.

The domain decomposition technique allows for replacing the singular perturbation in the form of the small hole $\mathbb{B}_{\rho}$ in the domain $\Omega_{\rho}$ by a regular perturbation in the truncated domain $\Omega_{R}$ on the boundary $\Gamma_{R}$. However, for this purpose elliptic theory is used only. This leads to a consideration of the asymptotic approximation which is 
of the first order with respect to a small parameter $\rho$. More precisely, the energy in the ring $\mathbb{B}_{R} \backslash \mathbb{B}_{\rho}$ for the Laplacian with non-homogeneous Dirichlet condition on $\Gamma_{R}$ and the homogeneous Neumann condition on $\Gamma_{\rho}$ is considered as a function of $\rho \rightarrow 0$. The asymptotic approximation of the energy is equivalent to the asymptotic approximation of the Steklov-Poincaré operator on $\Gamma_{R}$. In addition, we determine below the first term of order $\rho^{2}$ which turns out to be a bounded non-local operator on $L_{2}\left(\Gamma_{R}\right)$. The boundary condition imposed on $\Gamma_{R}$ reflects the presence of the defect in the ring $\mathbb{B}_{R} \backslash \mathbb{B}_{\rho}$. In this way, the presence of a singular perturbation in $\Omega_{\rho}$ is modeled by a regular perturbation of non-local boundary conditions on the boundary of truncated domain. The precision of such approximation for the hyperbolic evolution problems is still to be evaluated for some numerical examples.

Using the results of the Appendix we obtain the following expansion for the elliptic Steklov-Poincaré operator $A_{\rho}$ in the norm $\mathcal{L}\left(H^{1 / 2}\left(\Gamma_{R}\right), H^{-1 / 2}\left(\Gamma_{R}\right)\right)$ :

$$
A_{\rho}=A_{0}+\rho^{2} B+O\left(\rho^{4}\right)=A_{0}+\rho^{2} B+R_{\rho} .
$$

More specifically, $A_{0} \in \mathcal{L}\left(H^{1 / 2}\left(\Gamma_{R}\right) \mapsto H^{-1 / 2}\left(\Gamma_{R}\right)\right)$, the remainder $O\left(\rho^{4}\right)$ is uniformly bounded on bounded sets in the space $H^{1 / 2}\left(\Gamma_{R}\right)$ with values in $H^{-1 / 2}\left(\Gamma_{R}\right)$ and also can be considered as bounded from $H^{1}\left(\Gamma_{R}\right) \mapsto L_{2}\left(\Gamma_{R}\right)$. The operator $B$ is self-adjoint, from $H^{1 / 2}\left(\Gamma_{R}\right) \mapsto H^{-1 / 2}\left(\Gamma_{R}\right)$ and can be shown to be bounded operator $H^{1 / 2}\left(\Gamma_{R}\right) \mapsto L_{2}\left(\Gamma_{R}\right)$. The bounds are uniform in $\rho$.

The first term $A_{0}$ in the expansion of the operator $A_{\rho}$ is the Dirichlet to Neumann operator or Steklov-Poincaré operator of the ball, hence it is simply given by the standard Green formula for the ball $\mathbb{B}_{R}$. In other words, for a given function $w_{0}$ harmonic in $\mathbb{B}_{R}$, with the Dirichlet trace $v \in H^{1 / 2}\left(\Gamma_{R}\right)$, the value of the operator $A_{0}(v) \in H^{-1 / 2}\left(\Gamma_{R}\right)$ is just the Neumann trace of the harmonic function cf. (5).

The second term of the expansion in $\rho^{2}$ can be represented in two spatial dimensions in the equivalent form of the product of the line integrals over the circle $\Gamma_{R}=\{x:|x-\mathcal{O}|=R\}$ with the centre at the origin $\mathcal{O} .\langle B u, u\rangle$ is just the sum of squares of the line integrals, the trace on $\Gamma_{R}$ is integrated with polynomials of degree one in both space variables. The operator $B$ is self-adjoint since the bilinear form is symmetric

$$
\langle B u, u\rangle=b\left(\Gamma_{R} ; u, u\right)=-\frac{1}{2 \pi R^{6}}\left[\left(\int_{\Gamma_{R}} u x_{1} d s\right)^{2}+\left(\int_{\Gamma_{R}} u x_{2} d s\right)^{2}\right] .
$$

From the above representation, since the line integrals on $\Gamma_{R}$ are well defined for functions in $L_{2}\left(\Gamma_{R}\right)$, or even in $L_{1}\left(\Gamma_{R}\right)$, it follows that the operator $B$ can be extended to the bounded operator on $L_{2}\left(\Gamma_{R}\right)$,

$$
B \in \mathcal{L}\left(L_{2}\left(\Gamma_{R}\right) \mapsto L_{2}\left(\Gamma_{R}\right)\right)
$$

since the symmetric bilinear form of the operator, given by the equality

$$
\begin{aligned}
\langle B u, v\rangle & =b\left(\Gamma_{R} ; u, v\right) \\
& =-\frac{1}{2 \pi R^{6}}\left[\left(\int_{\Gamma_{R}} u x_{1} d s\right)\left(\int_{\Gamma_{R}} v x_{1} d s\right)+\left(\int_{\Gamma_{R}} u x_{2} d s\right)\left(\int_{\Gamma_{R}} v x_{2} d s\right)\right]
\end{aligned}
$$


is continuous for all $u, v \in L_{2}\left(\Gamma_{R}\right)$. In fact, the bilinear form

$$
L_{2}\left(\Gamma_{R}\right) \times L_{2}\left(\Gamma_{R}\right) \ni(u, v) \mapsto b\left(\Gamma_{R} ; u, v\right) \in \mathbb{R}
$$

is continuous with respect to the weak convergence since it has a simple structure

$$
b\left(\Gamma_{R} ; u, v\right)=l_{1}(u) l_{1}(v)+l_{2}(u) l_{2}(v), \quad u, v \in L_{1}\left(\Gamma_{R}\right)
$$

with two linear forms $v \mapsto l_{i}(v), i=1,2$, given by the line integrals on $\Gamma_{R}$. This gives us the additional regularity when replacing the singular perturbation of geometrical domain by the regular non-local perturbation $B$ of the non-local boundary operator $A_{\rho}$. The numerical results are required, however, to confirm if this regular approximation of the hole is robust, and efficient e.g., for the low frequencies, which seems to be the case on the strength of theoretical considerations. The presence of "hidden regularity" regularization is known to produce strong stability properties for approximations of boundary conditions $[5,13]$.

\subsection{Approximate model in $\Omega_{R}$}

For the domain $\Omega_{\rho}$ with defect in the form of a hole $\mathbb{B}_{\rho}$, the wave equation should be considered in the singularly perturbed domain with a small hole. Our aim is to consider a model with the domain without any hole, but with some influence of the defect modeled by means of the asymptotic analysis, cf. Appendix for the case of the energy functional and of the asymptotic analysis for the Steklov-Poincaré operator. The domain decomposition method consists in using the truncated domain $\Omega_{R}$ which contains no defect, however, the defect is modeled by a regular perturbation of the boundary conditions by the non-local Steklov-Poincaré operator. By the asymptotic analysis, the exact Steklov-Poincaré operator is approximated by its one term asymptotic approximation. Therefore, the approximate model in $\Omega_{R} \times(0, T)$ leads to the following hyperbolic equation with absorbing boundary conditions (where $\varepsilon>0$ is a fixed parameter corresponding to the regularization)

$$
\left.\begin{array}{ll}
\frac{\partial^{2} y}{\partial t^{2}}-\Delta y=f & \text { in } \Omega_{R} \times(0, T), \\
\frac{\partial y}{\partial \eta}+\varepsilon y_{t}=v & \text { on } \Gamma \times(0, T)=\Sigma, \\
\frac{\partial y}{\partial \eta}+\varepsilon y_{t}+A_{\rho}(y)=0 & \text { on } \Gamma_{R} \times(0, T)=\Sigma_{R}, \\
y(x, 0)=y_{0}(x) & \text { in } \Omega_{R}, \\
\frac{\partial y(x, 0)}{\partial t}=y_{1}(x) & \text { in } \Omega_{R} .
\end{array}\right\}
$$

We are interested in optimizing finite energy solutions $\left(y, y_{t}\right) \in C\left(H^{1}\left(\Omega_{R}\right) \times\right.$ $L_{2}\left(\Omega_{R}\right)$ ) by means of boundary control $v \in L_{2}(\Sigma)$. We shall show that for the associated optimal control problem, the solutions are stable with respect to the small 
parameter $\rho \rightarrow 0$. In order to make the meaning of "finite energy" solutions to (11) precise, we shall define weak solutions via standard variational equality

$$
\begin{aligned}
& \frac{d}{d t}\left(y_{t}, \phi\right)_{\Omega_{R}}+(\nabla y, \nabla \phi)_{\Omega_{R}}+\left(\varepsilon y_{t}-v, \phi\right)_{\Gamma} \\
& \quad+\left(\varepsilon y_{t}, \phi\right)_{\Gamma_{R}}+\left(A_{\rho}^{1 / 2} y, A_{\rho}^{1 / 2}\right)_{\Gamma_{R}}=0, \quad \forall \phi \in H^{1}\left(\Omega_{R}\right)
\end{aligned}
$$

with the initial conditions $Y(0)=\left(y_{0}, y_{1}\right) \in H^{1}\left(\Omega_{R}\right) \times L_{2}\left(\Omega_{R}\right)$.

Remark 1 The presence of the parameter $\varepsilon>0$ in both boundary conditions provides for "hidden regularity" effect on boundary traces of solutions. It will be shown that finite energy solutions satisfy $\left.y_{t}\right|_{\Gamma \cup \Gamma_{R}} \in L_{2}\left(\Sigma \cup \Sigma_{R}\right)$. Thus, the boundary terms involving time derivatives restricted to the boundary are well defined in the definition of weak solution given in (12).

As already mentioned before, this type of "hidden regularization" regularization has been used in the context of FEM approximations of Riccati solutions [5, 20] and in the context of domain decomposition [13].

\section{Neumann control problem in $U=L_{2}(\Gamma \times(0, T))$. Main results}

We consider the following optimal boundary control problem defined in domain $\Omega_{R}$. Let $U=L_{2}(\Gamma \times(0, T))$ be the space of controls. The time horizon $T$ is fixed and the parameter of regularization $\varepsilon>0$.

With $Y \equiv\left[y, y_{t}\right]$, a solution to (11), and

$$
H \equiv H^{1}(\Omega) \times L_{2}(\Omega), \quad \mathcal{R} \in \mathcal{L}(H)
$$

the functional cost is given by

$$
I(v)=\frac{1}{2}\left|\mathcal{R}\left(Y(T ; v)-Y_{d}\right)\right|_{H}^{2}+\frac{\alpha}{2} \int_{0}^{T} \int_{\Gamma}|v|^{2} d s d t .
$$

The following constraints are imposed on the controls $v \in U_{a d}$ :

$$
U_{a d}=\left\{v \in L_{2}(\Gamma \times(0, T)), 0 \leq v(x, t) \leq 1\right\} .
$$

Our first result pertains to existence and regularity of optimal pair $\left(v_{\rho}^{0}, Y_{\rho}^{0}\right)$ corresponding to optimal control problem consisting of minimizing the functional (13) subject to (14) and (11).

Theorem 1 For all initial data $Y(0) \in H$, terminal data $Y_{d} \in H$, right-hand side $f \in L_{1}\left(0, T ; L_{2}\left(\Omega_{R}\right)\right)$, and all $\rho>0$ there exists a unique optimal control $v_{\rho}^{0} \in$ $L_{2}(\Sigma) \cap U_{a d}$ and such that optimal state $Y_{\rho}^{0} \in C([0, T] ; H)$. In addition, for $\varepsilon>0$, the following boundary regularity holds: $\left.\frac{d}{d t} y_{\rho}^{0}\right|_{\partial \Omega_{R}} \in L_{2}\left(\Sigma \cup \Sigma_{R}\right)$. 
Remark 2 We note that the boundary regularity of the velocity does not follow from interior regularity. This is an independent trace regularity result which turns out to be essential in characterizing optimal control.

When $\varepsilon=0$ one can still deduce existence of optimal control and optimal trajectory, however the boundary regularity of solutions fails. Since this regularity is critical for further sensitivity analysis, we shall henceforth be assuming that $\varepsilon>0$.

Our GOAL: is the asymptotic analysis of the optimal control when $\rho \rightarrow 0$. In reference to problem (11), the following result on the stability of optimal controls for $\rho \rightarrow 0$ holds.

Theorem 2 Let us consider the minimization of the cost functional (13) evaluated for the state equation (11) with $f=0$ and initial-terminal data in $H=H^{1}\left(\Omega_{R}\right) \times$ $L_{2}\left(\Omega_{R}\right)$, subject to the control constraints (14). Then the unique optimal control $u_{\rho}^{0}$ admits for $\rho \rightarrow 0$ the one term asymptotics

$$
u_{\rho}=u+\rho^{2} q+o\left(\rho^{2}\right) \quad \text { in } L_{2}(\Gamma \times(0, T)) \text {, }
$$

where $q$ is given by a unique solution of the auxiliary optimal control problem ( $c f$. Lemma 8) with the state equation (63), the cost functional (57), and the set of admissible controls (61).

The remaining part of the paper is devoted to the proof of the main theorem.

\section{Existence and regularity theory}

In this section we study forward regularity properties of solutions to the initialboundary value problem given in (11).

\section{Theorem 3 Regularity theorem}

Let

- $f \in L_{1}\left(0, T ; L_{2}\left(\Omega_{R}\right)\right), v \in L_{2}\left(0, T ; L_{2}(\Gamma)\right)$

- $y_{0} \in H^{1}\left(\Omega_{R}\right), y_{1} \in L_{2}\left(\Omega_{R}\right)$.

Then, there exists a unique solution of the state equation in the truncated domain

$$
y \in C\left(0, T ; H^{1}\left(\Omega_{R}\right)\right) \cap C^{1}\left(0, T ; L_{2}\left(\Omega_{R}\right)\right)
$$

and such that the following hidden regularity holds:

$$
\begin{aligned}
&\left.y\right|_{\Gamma_{R}} \in H^{1}\left(\Sigma_{R}\right) \cap C\left(0, T ; H^{1 / 2}\left(\Gamma_{R}\right)\right) . \\
&\left.y\right|_{\Gamma} \in H^{1}(\Sigma) .
\end{aligned}
$$


In addition, the following bound is available for all $0 \leq t \leq T$ :

$$
\begin{aligned}
& |y(t)|_{1, \Omega_{R}}^{2}+\left|y_{t}(t)\right|_{0, \Omega_{R}}^{2}+2 \varepsilon \int_{0}^{t}\left|y_{t}\right|_{\Gamma}^{2} d s+2 \varepsilon \int_{0}^{t}\left[\left|y_{t}\right|_{\Gamma_{R}}^{2}+|y|_{H^{1}\left(\Gamma_{R} \cup \Gamma\right)}^{2}\right] d s \\
& \quad \leq C\left[|y(0)|_{1, \Omega_{R}}^{2}+\left|y_{t}(0)\right|_{0, \Omega_{R}}^{2}\right]+\frac{c}{\varepsilon}\|v\|_{L_{2}(\Sigma)}^{2},
\end{aligned}
$$

where the constants are uniform in $\rho \geq 0$.

Proof We shall approach the problem of existence of solutions to (30)-or equivalently to its variational form defined in (12) - by using semigroup theory. In fact, it is enough to show that the semigroup solutions generated by differential equation (30) have desired boundary regularity. This allows to obtain variational formulation by taking strong limits of strong semigroup solutions defined on $H$. To this aim we define several operators. Let $A_{N}$ denotes the Laplacian with zero Neumann boundary data on $\partial \Omega_{R}$. This is to say $A_{N}: D\left(A_{N}\right) \subset L_{2}\left(\Omega_{R}\right) \mapsto L_{2}\left(\Omega_{R}\right)$ is defined by

$$
A_{N} u=-\Delta u, \quad u \in D\left(A_{N}\right) \equiv\left\{u \in H^{2}\left(\Omega_{R}\right): \frac{\partial}{\partial \nu} u=0 \text { on } \Gamma \cup \Gamma_{R}\right\}
$$

Let $N$ (resp. $N_{R}$ ) denote the Neumann harmonic extension from $\Gamma$ (resp. $\Gamma_{R}$ ) into $\Omega_{R}$. This is to say $N: L_{2}(\Gamma) \mapsto L_{2}\left(\Omega_{R}\right)$ is defined by $w \equiv N v$ iff $\Delta w=0$ in $\Omega_{R}$ and $\frac{\partial}{\partial v} w=v$ on $\Gamma, \frac{\partial}{\partial v} w=0$ on $\Gamma_{R}$.

Similarly, $N_{R}: L_{2}\left(\Gamma_{R}\right) \mapsto L_{2}\left(\Omega_{R}\right)$ is defined by $w \equiv N v$ iff $\Delta w=0$ in $\Omega_{R}$ and $\frac{\partial}{\partial v} w=0$ on $\Gamma, \frac{\partial}{\partial v}=v$ on $\Gamma_{R}$.

In defining Neumann harmonic extensions, without loss of generality we may assume that zero eigenvalue is mode out. This has no effect on further analysis, since eventually

$$
\left|A_{N}^{1 / 2} u\right|_{\Omega_{R}}^{2}+\left|A_{\rho}^{1 / 2} u\right|_{\Gamma_{R}}^{2} \sim|u|_{H^{1}\left(\Omega_{R}\right)}^{2},
$$

so the static elliptic operator controls full $H^{1}$ norm.

The abstract second order form of the problem under consideration (see $[15,20])$ is the following:

$$
\begin{aligned}
y_{t t} & +A_{N} y+\varepsilon A_{N} N N^{*} A_{N} y_{t}+\varepsilon A_{N} N_{R} N_{R}^{*} A_{N} y_{t}+A_{N} N_{R} A_{\rho}(y) \\
& =f+A_{N} N v
\end{aligned}
$$

with the initial conditions $y_{0} \in H^{1}\left(\Omega_{R}\right), y_{1} \in L_{2}\left(\Omega_{R}\right)$.

The above representation uses the following identifications:

$$
\begin{aligned}
& N^{*} A_{N} y=\left.y\right|_{\Gamma}, \quad y \in H^{1}\left(\Omega_{R}\right), \\
& N_{R}^{*} A_{N} y=\left.y\right|_{\Gamma_{R}}, \quad y \in H^{1}\left(\Omega_{R}\right),
\end{aligned}
$$

which follow from the application of Green's formula [15].

STEP 1. We shall prove that (11) with $v=0, f=0$ generates a strongly continuous semigroup on $H^{1}\left(\Omega_{R}\right) \times L_{2}\left(\Omega_{R}\right)$. In order to accomplish this, we find it convenient 
to topologize $H$ with equivalent norm given by

$$
\begin{aligned}
& |y|_{H}^{2} \equiv\left|A_{N}^{1 / 2} y_{1}\right|_{\Omega_{R}}^{2}+\left|A_{0}^{1 / 2}\left(y_{1}\right)\right|_{\Gamma_{R}}^{2}+\left|y_{2}\right|_{\Omega_{R}}^{2}, \\
& |y|_{H_{\rho}}^{2} \equiv\left|A_{N}^{1 / 2} y_{1}\right|_{\Omega_{R}}^{2}+\left|A_{\rho}^{1 / 2}\left(y_{1}\right)\right|_{\Gamma_{R}}^{2}+\left|y_{2}\right|_{\Omega_{R}}^{2} .
\end{aligned}
$$

In view of Lemma 1 these are equivalent norms to the standard $H^{1}\left(\Omega_{R}\right) \times L_{2}\left(\Omega_{R}\right)$ norms. The inner product generated by $H$ is the following

$$
(y, w)_{H_{\rho}} \equiv\left(A_{N}^{1 / 2} y_{1}, A_{N}^{1 / 2} w_{1}\right)_{\Omega_{R}}+\left(A_{\rho}^{1 / 2} y_{1}, A_{\rho}^{1 / 2} w_{1}\right)_{\Gamma_{R}}+\left(y_{2}, w_{2}\right)_{\Omega_{R}} .
$$

We introduce the operator $\mathcal{A}_{\rho}: H \mapsto H$ whose action is defined by

$$
\mathcal{A}_{\rho}\left(y_{1}, y_{2}\right) \equiv\left(\begin{array}{c}
0 \\
I \\
-A_{N}-A_{N} N_{R} A_{\rho} N_{R}^{*} A_{N}-\varepsilon A_{N} N_{R} N_{R} A_{N}^{*}-\varepsilon A_{N} N N^{*} A_{N}
\end{array}\right) .
$$

It follows that for $y=\left(y_{1}, y_{2}\right) \in D\left(\mathcal{A}_{\rho}\right)$, where $D\left(\mathcal{A}_{\rho}\right)$ is the maximal domain, we obtain:

$$
\begin{aligned}
\left(\mathcal{A}_{\rho} y, y\right)_{H_{\rho}} & \\
= & -\varepsilon\left|N^{*} A_{N} y_{2}\right|_{\Gamma}^{2}-\varepsilon\left|N_{R}^{*} A_{N} y_{2}\right|_{\Gamma_{R}}^{2}-\left(A_{N} N A_{\rho} N_{R}^{*} A_{N} y_{1}, y_{2}\right)_{\Omega_{R}} \\
& -\left(A_{N} y_{1}, y_{2}\right)+\left(A_{\rho}^{1 / 2} N_{R}^{*} A_{N} y_{1}, A_{\rho}^{1 / 2} N_{R}^{*} A_{N} y_{2}\right)_{\Omega_{R}}+\left(A_{N}^{1 / 2} y_{1}, A_{N}^{1 / 2} y_{2}\right)_{\Omega_{R}} \\
= & -\varepsilon\left|N^{*} A_{N} y_{2}\right|_{\Gamma}^{2}-\frac{1}{2} \varepsilon\left|N_{R}^{*} A_{N} y_{2}\right|_{\Gamma_{R}}^{2}+C_{\varepsilon}|y|_{H}^{2}
\end{aligned}
$$

where we have used inner product defined on $H_{\rho}$. This gives that $\mathcal{A}_{\rho}$ is dissipative.

In order to prove the generation of the semigroup we need to establish maximal dissipativity. This is done as follows:

Maximal dissipativity, by Minty's Theorem, is equivalent to the range conditions, i.e. solvability of

$$
\mathcal{A}_{\rho} y-y=f \in H
$$

for every $f \in H$. Writing in the coordinates

$$
\begin{aligned}
& y_{2}-y_{1}=f_{1} \in H^{1}\left(\Omega_{R}\right), \\
& A_{N} y_{1}+A_{N} N_{R} A_{\rho} N_{R}^{*} A_{N} y_{1}+y_{2}+\varepsilon A_{N} N_{R} N_{R}^{*} A_{N} y_{2}+\varepsilon A_{N} N N^{*} A_{N} y_{2} \\
& \quad=f_{2} \in L_{2}\left(\Omega_{R}\right),
\end{aligned}
$$

which is equivalent to

$$
\begin{aligned}
& A_{N} y_{1}+A_{N} N_{R} A_{\rho} N_{R}^{*} A_{N} y_{1}+y_{1}+\varepsilon A_{N} N_{R} N_{R}^{*} A_{N} y_{1}+\varepsilon A_{N} N N^{*} A_{N} y_{1} \\
& \quad=-f_{1}-f_{2}-\varepsilon A_{N} N_{R} N_{R}^{*} A_{N} f_{1}-\varepsilon A_{N} N N^{*} A_{N} f_{1} .
\end{aligned}
$$

The operator

$$
\mathcal{B} \equiv A_{N}+A_{N} N_{R} A_{\rho} N_{R}^{*} A_{N}+I+\varepsilon A_{N} N_{R} N_{R}^{*} A_{N}+\varepsilon A_{N} N N^{*} A_{N}
$$


is a Lax-Milgram operator on the space $V \equiv H^{1}\left(\Omega_{R}\right)$. Indeed,

$$
\begin{aligned}
(\mathcal{B} u, u)_{L_{2}\left(\Omega_{R}\right)}= & \left|A_{N}^{1 / 2} u\right|_{\Omega_{R}}^{2}+\left|A_{\rho}^{1 / 2} N_{R}^{*} A_{N} u\right|_{\Gamma_{R}}^{2}+|u|_{\Omega_{R}}^{2} \\
& +\varepsilon\left|N^{*} A_{N} y\right|_{\Gamma}^{2}+\varepsilon\left|N_{R}^{*} A_{N} u\right|_{\Gamma_{R}}^{2} \geq|u|_{1, \Omega_{R}}^{2} .
\end{aligned}
$$

The continuity of the associated form follows by Lemma 1 .

Since with $f \in H^{1}\left(\Omega_{R}\right)$, and $R(N) \subset D\left(A_{N}^{\alpha}\right)$ for $0 \leq \alpha<3 / 4$, we have

$$
A_{N} N_{R} N_{R}^{*} A_{N} f_{1} \in\left[D\left(A_{N}^{1 / 2}\right)\right]^{\prime} \subset V^{\prime}
$$

and

$$
A_{N} N N^{*} A_{N} f_{1} \in\left[D\left(A_{N}^{1 / 2}\right)\right]^{\prime} \subset V^{\prime} .
$$

The above leads to the solvability of (19). Thus, the generation of a strongly continuous semigroup is deduced via monotone operator theory.

Remark 3 We note that maximal dissipativity property of the operator $\mathcal{A}_{\rho}$ does not depend on strict positivity of the parameter $\varepsilon$. Thus, the conclusion on the existence of semigroup solution is independent on the regularization. However, in order to prove the energy inequality, as stated in the Theorem 3 , the presence of $\varepsilon>0$ is critical. This provides for additional boundary regularity.

In order to prove the energy inequality stated in Theorem 3, we first apply the energy-multipliers method. Taking first $v=0$ and multiplying (11) by $y_{t}$ gives the following energy equality:

$$
|Y(t)|_{H_{\rho}}^{2}+\varepsilon \int_{0}^{t}\left|N_{R}^{*} A_{N} y_{t}\right|_{\Gamma_{R}}^{2}+\varepsilon \int_{0}^{t}\left|N^{*} A_{N} y_{t}\right|_{\Gamma}^{2}=|Y(0)|_{H_{\rho}}^{2} .
$$

Since $\left.y\right|_{\Gamma_{R} \cup \Gamma} \in H^{1}\left(0, T ; L_{2}\left(\Gamma_{R}\right)\right), Y \in C(0, T ; H)$ and $\frac{\partial}{\partial v} y \in L_{2}(\Sigma)$, hidden regularity applies $[16,21]$ and implies the $L_{2}$ regularity of tangential derivatives on the boundary $\Gamma$. The same applies to $\Gamma_{R}$ after taking into considerations the regularity for "small frequencies" exhibited by elliptic problem resulting from microlocalization of the wave operator to "small" time dual variables (frequencies). This leads to consideration of elliptic problem (microlocally) which are driven by $L_{2}$ internal force and $L_{2}$ boundary data (see [18]). This is to say that microlocal solutions satisfy

$$
\Delta y=f \in L_{2}(\Omega), \quad \frac{\partial}{\partial v} y=h \quad \text { on } \Gamma, \quad \frac{\partial}{\partial v} y+A_{\rho} y=g \quad \text { on } \Gamma_{R}
$$

display the regularity:

$$
|y|_{1+s, \Omega_{R}}+|y|_{H^{1 / 2+s}\left(\Gamma \cup \Gamma_{R}\right)} \leq C\left(|f|_{L_{2}\left(\Omega_{R}\right)}+|h|_{-1 / 2+s, \Gamma}+|g|_{-1 / 2+s, \Gamma_{R}}\right)
$$

for all $s \in[0,1 / 2]$ uniformly in the parameter $\rho>0$. Applying the above inequality with $s=1 / 2$ and accounting for the fact that we already know that $y_{t} \in L_{2}\left(\Sigma_{R} \cup \Sigma\right)$ 
gives:

$$
\int_{0}^{T}\left|\nabla_{\Gamma_{R}} y\right|_{\Gamma_{R}}^{2}+\left.|\nabla|_{\Gamma} y\right|_{\Gamma} ^{2} d s \leq c \varepsilon^{-1}|Y(0)|_{H_{\rho}}^{2} .
$$

By Lemma 1 we have that $|Y|_{H_{\rho}} \sim|Y|_{H}$, uniformly in $\rho \in[0,1]$, so the norms $H_{\rho}$ can be replaced (with appropriate change of the constants) by norms in $H$. This provides the desired bound with $v=0$.

STEP 2: We go back to the main equation (15) which is the boundary perturbation of the $\omega$ dissipative semigroup. As such, it can be written as $Y \equiv\left[y, y_{t}\right]$

$$
\frac{d}{d t} Y=\mathcal{A}_{\rho} Y+\left(\begin{array}{c}
0 \\
A_{N} N_{R} v+f
\end{array}\right) .
$$

It is at this point where absorbing damping on the boundary $\Gamma$ is critical. (Absorbing damping on $\Gamma_{R}$ will be needed for sensitivity analysis).

Indeed, multiplying (15) by $y_{t}$ and integrating by parts (this procedure is formally applied to smooth approximations of the problem and followed by limit process [20]) yields:

$$
\begin{aligned}
& |Y(t)|_{H_{\rho}}^{2}+2 \varepsilon \int_{0}^{t}\left|N^{*} A_{N} y_{t}\right|_{\Gamma}^{2} d s+2 \varepsilon \int_{0}^{t}\left|N_{R}^{*} A_{N} y_{t}\right|_{\Gamma_{R}}^{2} d s \\
& \quad \leq|Y(0)|_{H_{\rho}}^{2}+\int_{0}^{t}|f|_{\Omega_{R}}\left|y_{t}\right|_{\Omega_{R}} d s+\int_{0}^{t}\left(A_{N} N v, y_{t}\right)_{\Omega_{R}} d s .
\end{aligned}
$$

The above implies:

$$
\begin{aligned}
& \left|A_{N}^{1 / 2} y(t)\right|_{\Omega_{R}}^{2}+\left|y_{t}(t)\right|_{\Omega_{R}}^{2}+2 \varepsilon \int_{0}^{t}\left|N^{*} A_{N} y_{t}\right|_{\Gamma}^{2} d s+2 \varepsilon \int_{0}^{t}\left|N_{R}^{*} A_{N} y_{t}\right|_{\Gamma_{R}}^{2} d s \\
& \leq C\left|A_{N}^{1 / 2} y(0)\right|_{\Omega_{R}}^{2}+C\left|y_{t}(0)\right|_{\Omega_{R}}^{2}+1 / 2 \sup _{s \in[0, t]}\left|y_{t}(s)\right|_{\Omega_{R}}^{2} \\
& \quad+C\left(\int_{0}^{t}|f|_{\Omega_{R}} d s\right)^{2}+\varepsilon \int_{0}^{t}\left|N^{*} A_{N} y_{t}\right|_{\Gamma}^{2} d s+\frac{2}{\varepsilon} \int_{0}^{t} \int_{\Gamma}|v|^{2} d x d s .
\end{aligned}
$$

Hence

$$
\begin{aligned}
& |Y(t)|_{H}^{2}+\varepsilon \int_{0}^{t}\left|y_{t}\right|_{\Gamma_{R}}^{2}+\varepsilon \int_{0}^{t}\left|y_{t}\right|_{\Gamma}^{2} \\
& \quad \leq C_{t}\left[|Y(0)|_{H}^{2}+C\left(\int_{0}^{t}|f|_{\Omega_{R}} d s\right)^{2}+\frac{c}{\varepsilon} \int_{0}^{t} \int_{\Gamma}|v|^{2} d x d s\right] .
\end{aligned}
$$

Since $\left.y_{t}\right|_{\Gamma_{R}} \in L_{2}\left(\Sigma_{R}\right)$ and solutions are of finite energy, hidden regularity [16] along with elliptic estimate (20) gives the control of all tangential-time and space derivatives on the boundary. This completes the proof of Theorem 3 .

It is convenient to introduce the following notation: 
- $S_{\rho, t}: H \mapsto H$ is the semigroup generated by (11) with $f=0, v=0$.

- $\left(L_{\rho} u\right)(t)=\int_{0}^{t} S_{\rho, t-s} D u(s) d s$ where $D u \equiv\left[0, A_{N} N u\right]$, or equivalently solution to (11) with $f=0, y_{0}, y_{1}=0, v=u$. The bounds are independent on $\rho$.

Theorem 3 implies for all $t \in[0, T]$.

\section{Corollary 1}

1. $\left|S_{\rho, t} Y\right|_{H} \leq C_{t}|Y|_{H}$

2. $\varepsilon \int_{0}^{T}\left[\left|\gamma S_{\rho, t} Y\right|_{L_{2}(\Gamma)}^{2}+\left|\gamma_{R} S_{\rho, t} Y\right|_{L_{2}\left(\Gamma_{R}\right)}^{2}\right] \leq C|Y|_{H}^{2}$

3. $\left|L_{\rho}(u)\right|_{C(0, T, H)} \leq \frac{C}{\sqrt{\varepsilon}}|u|_{L_{2}(\Sigma)}$

4. $\left|\gamma P_{2} L_{\rho} u\right|_{L_{2}(\Sigma)}+\left|\gamma_{R} P_{1} L_{\rho} u\right|_{H^{1}\left(\Sigma_{R} \cup \Sigma\right)} \leq \frac{C}{\varepsilon}|u|_{L_{2}(\Sigma)}$

where the bounds are uniform in $\rho \geq 0$ and also $t \in[0, T]$.

\section{Optimal control problem}

We shall analyze next the optimal boundary control problem (11)-(13) in the domain $\Omega_{R}$ with the fixed parameter $\rho>0$. The solution of the formulated optimal control problem is equivalent to seeking a $v_{\rho}^{0} \in U_{a d}$ such that $I\left(v_{\rho}^{0}\right) \leq I(v)$ for all $v \in U_{a d}$. Standard arguments in calculus of variations lead to the following results for $\alpha>0$ a unique optimal control $v_{\rho}^{0}$ is characterized by the following condition

$$
I^{\prime}\left(v_{\rho}^{0}\right)\left(v-v_{\rho}^{0}\right) \geq 0 \quad \forall v \in U_{a d} .
$$

Using the form of the performance functional (13) we can express (24) in the following form:

$$
\begin{aligned}
& \left(\mathcal{R}^{*} \mathcal{R}\left(Y\left(x, T ; v_{\rho}^{0}\right)-Y_{d}\right),\left(Y(x, T ; v)-Y\left(x, T ; v_{\rho}^{0}\right)\right)_{H}\right. \\
& \quad+\frac{\alpha}{2} \int_{0}^{T} \int_{\Gamma} v_{\rho}^{0}\left(v-v_{\rho}^{0}\right) d x d t \geq 0 \quad \forall v \in U_{a d} .
\end{aligned}
$$

After denoting $L_{\rho, T} u=L_{\rho} u(T)$ and observing that $Y(T, v)=S_{\rho, T} Y_{0}+L_{\rho, T} v$ (25) gives:

$$
\begin{gathered}
\int_{0}^{T}\left(L_{\rho, T}^{*} \mathcal{R}^{*} \mathcal{R}\left(L_{\rho, T} v_{\rho}^{0}+S_{\rho, T} Y_{0}-Y_{D}\right), v-v_{\rho}^{0}\right)_{\Gamma} d t \\
+\frac{\alpha}{2} \int_{0}^{T}\left(v_{\rho}^{0}, v-v_{\rho}^{0}\right)_{\Gamma} d t \geq 0 \quad \forall v \in U_{a d},
\end{gathered}
$$

which provides the following characterization of the optimal control.

$$
v_{\rho}^{0} \equiv P_{U_{a d}}\left[\frac{-1}{\alpha} L_{\rho, T}^{*} \mathcal{R}^{*} \mathcal{R}\left(L_{\rho, T} v_{\rho}^{0}+S_{\rho, T} Y_{0}-Y_{D}\right)\right]=P_{U_{a d}}\left[-C_{\rho} v_{\rho}^{0}+F_{\rho}\right],
$$

where

$$
C_{\rho} \equiv \frac{1}{\alpha} L_{\rho, T}^{*} \mathcal{R}^{*} \mathcal{R} L_{\rho, T}, \quad F_{\rho} \equiv \frac{-1}{\alpha} L_{\rho, T}^{*} \mathcal{R}^{*} \mathcal{R}\left(S_{\rho, T} Y_{0}-Y_{D}\right)
$$


Here we use the topology of $H$ (independent on the parameter $\rho$ ) for the computations of the adjoints.

Direct calculation of the adjoint operator $L_{\rho, T}^{*}$ yields:

$$
L_{\rho, T}^{*} \Phi \equiv \gamma \Psi_{2}(t), \quad \Psi(t) \equiv S_{\rho}^{*}(T-t) \Phi .
$$

In order to calculate the adjoint explicitly we find useful to introduce the notation

$$
\tilde{A}_{N} \equiv A_{N}+A_{N} N_{R} A_{0} \gamma_{R}=A_{N}+A_{N} N_{R} A_{0} N_{R}^{*} A_{N}
$$

This is self-adjoint, positive operator acting on $L_{2}\left(\Omega_{R}\right)$ and $\left|\tilde{A}_{N}^{1 / 2} u\right|_{\Omega_{R}} \sim|u|_{1, \Omega_{R}}$. We also note that

$$
|Y|_{H}^{2}=\left|\tilde{A}_{N}^{1 / 2} y_{1}\right|_{\Omega_{R}}^{2}+\left|y_{2}\right|_{\Omega_{R}}^{2} .
$$

With the above notation we have

$$
\mathcal{A}_{\rho}^{*}\left(y_{1}, y_{2}\right)=\left[-y_{2}-\tilde{A}_{N}^{-1} A_{N} N_{R} R_{\rho}^{*} \gamma_{R} y_{2}, \tilde{A}_{N} y_{1}-\varepsilon A_{N} N_{R} \gamma_{R} y_{2}-\varepsilon A_{N} N \gamma y_{2}\right]
$$

where $R_{\rho}$ is introduced in (8).

To simplify (25), we introduce the adjoint equation. For every $v \in U_{a d}$, we define the adjoint variable

$$
P_{\rho}(t)=\left[p_{\rho, 1}(t), p_{\rho, 2}(t)\right] \equiv S_{\rho, T-t}^{*} \mathcal{R}^{*} \mathcal{R}\left(Y^{0}(T)-Y_{d}\right) .
$$

We verify that the vector $P_{\rho}$ is the solution of the following system of equations:

$$
\begin{aligned}
\frac{d}{d t} p_{\rho, 1} & =p_{\rho, 2}+{\tilde{A_{N}}}^{-1} A_{N} N_{R} R_{\rho}^{*} \gamma_{R} p_{\rho, 2}, \\
\frac{d}{d t} p_{\rho, 2} & =-\tilde{A}_{N} p_{\rho, 1}+\varepsilon A_{N} N_{R} \gamma_{R} p_{\rho, 2}+\varepsilon A_{N} N \gamma p_{\rho, 2} .
\end{aligned}
$$

The above system can be rewritten as the following PDE-non-local system

$$
\begin{aligned}
& \frac{d}{d t} p_{\rho, 1}=p_{\rho, 2}+{\tilde{A_{N}}}^{-1} A_{N} N_{R} R_{\rho}^{*} \gamma_{R} p_{\rho, 2}, \quad \frac{d}{d t} p_{\rho, 2}=\Delta p_{\rho, 1} \quad \text { in } \Omega_{R} \times(0, T), \\
& \frac{\partial p_{\rho, 1}}{\partial \eta}-\varepsilon p_{\rho, 2}=0 \\
& \text { on } \Gamma \times(0, T) \text {, } \\
& \frac{\partial p_{\rho, 1}}{\partial \eta}-\varepsilon p_{\rho, 2}+A_{0}\left(p_{\rho, 1}\right)=0 \\
& \text { on } \Gamma_{R} \times(0, T) \text {, } \\
& P_{\rho}(x, T ; v)=\mathcal{R}^{*} \mathcal{R}\left(Y_{\rho}^{0}(T)-Y_{d}\right), \\
& \text { in } \Omega_{R} \text {, }
\end{aligned}
$$

where $Y_{\rho}^{0}$ is the optimal trajectory corresponding to $\rho$ problem.

Remark 4 We note that for $\rho=0$, we have $R_{\rho}=0$ and the adjoint equation for the variable $P^{0}(t)=\left[p^{0}(t), p_{t}^{0}(t)\right]$ can be written as: 


$$
\begin{aligned}
p_{t t}^{0} & =\Delta p^{0}, \\
\frac{\partial}{\partial v} p^{0}-\varepsilon p_{t}^{0} & =0 \quad \text { on } \Gamma, \\
\frac{\partial}{\partial v} p^{0}-\varepsilon p_{t}^{0}+A_{0}\left(p^{0}\right) & =0 \quad \text { on } \Gamma_{R}, \\
P^{0}(T) & =\mathcal{R}^{*} \mathcal{R}\left(Y^{0}(T)-Y_{d}\right) .
\end{aligned}
$$

Lemma 2 The following estimate holds for solutions to (30) for all $t \in[0, T]$.

$$
\begin{aligned}
& \left|p_{\rho, 1}(t)\right|_{1, \Omega_{R}}^{2}+\left|p_{\rho, 2}(t)\right|_{0, \Omega_{R}}^{2}+\varepsilon \int_{t}^{T}\left|p_{\rho, 2}\right|_{\Gamma}^{2} \\
& \quad+\varepsilon \int_{t}^{T}\left|p_{\rho, 2}\right|_{\Gamma_{R}}^{2} \leq C_{t}\left(\left|p_{\rho, 1}(T)\right|_{1, \Omega_{R}}^{2}+\left|p_{\rho, 2}(T)\right|_{0, \Omega_{R}}^{2}\right) .
\end{aligned}
$$

Proof This result follows from the same arguments as used in the proof of Theorem 3. In order to obtain the energy estimate we multiply the first equation in (29) by $\tilde{A}_{N} p_{1}$, the second equation by $p_{2}$ and use duality pairings. This gives:

$$
\left|P_{\rho}(t)\right|_{H}^{2}+\varepsilon \int_{t}^{T}\left|p_{2, \rho}\right|_{\Gamma \cup \Gamma_{R}}^{2} d s=\left|P_{\rho}(T)\right|_{H}^{2}+\int_{t}^{T}\left(R_{\rho}^{*} \gamma_{R} p_{\rho, 2}, \gamma_{R} p_{1, \rho}\right)_{\Gamma_{R}} d s
$$

Since $R_{\rho}^{*}: L_{2}\left(\Gamma_{R}\right) \mapsto H^{-1 / 2}\left(\Gamma_{R}\right)$ is bounded uniformly in $\rho$, we obtain

$$
\begin{aligned}
& \left(R_{\rho}^{*} \gamma_{R} p_{\rho, 2}, \gamma_{R} p_{1, \rho}\right)_{\Omega_{R}} \\
& \quad \leq C\left|p_{1}\right|_{1, \Omega_{R}}\left|\gamma_{R} p_{2}\right|_{0, \Gamma_{R}} \leq C_{\varepsilon}\left|p_{1}\right|_{1, \Omega_{R}}^{2}+1 / 4 \varepsilon\left|\gamma_{R} p_{2}\right|_{0, \Gamma_{R}}^{2} .
\end{aligned}
$$

The above estimate leads, via Gronwall's inequality, to the final conclusion in Lemma 2.

Remark 5 One could prove additional tangential regularity of $\left.p_{\rho, 1}\right|_{\Gamma_{R}} \in H^{1}\left(\Gamma_{R}\right)$ for $\rho$ sufficiently small, which would allow to relax regularity of $R_{\rho}^{*}: L_{2} \mapsto H^{-1}$. This step, however, would require analysis similar to that given in Regularity Theorem 3 but applied to $p_{\rho, 1}$ equation and followed by perturbation argument in order to incorporate non-local operator on the right side of the first equation in (30). It is for this point where smallness of $\rho$ will be needed. Since this point is not essential, we shall not insist on the additional technicalities.

Theorem 4 Let the hypothesis of Theorem 1 be satisfied. Then for given $Y_{d}, Y_{0} \in H$, $v_{\rho}^{0} \in U_{a d}$, there exists a unique solution to (30)

$$
P_{\rho}\left(v_{\rho}\right)=\left[p_{\rho}\left(v_{\rho}^{0}\right), p_{\rho, t}\left(v_{\rho}^{0}\right)\right] \in C(H)
$$

and such that $\left.p_{\rho, 2}^{0}\right|_{\partial \Omega_{R}} \in L_{2}\left(\Sigma \cup \Sigma_{R}\right)$. 
We simplify (25) using the adjoint equation (30). This leads to:

$$
\int_{0}^{T} \int_{\Gamma}\left(p_{\rho, 2}+\alpha v_{\rho}^{0}\right)\left(v-v_{\rho}^{0}\right) d x d t \geq 0 \quad \forall v \in U_{a d} .
$$

Theorem 5 (Optimality Theorem) For the problem (11) with the performance functional (13) with $Y_{d} \in H$ and $\alpha>0$, and with constraints on the control (14), there exists a unique optimal control $v_{\rho}^{0}$ which satisfies the maximum condition (34). Moreover, $v_{\rho}^{0}=P_{U_{a d}}\left(-\frac{1}{\alpha} p_{\rho, 2}\right)$ where $P_{U_{a d}}$ is a projection operator on $U_{a d}$ with respect to $L_{2}$ topology.

Remark 6 Note, that the boundary regularity of the adjoint variable $p_{\rho, 2} \mid \Gamma \cup \Gamma_{R}$ represents hidden regularity of the solutions to the adjoint equation. This is critical in characterizing the optimal solution.

Remark 7 By using inner product induced by $A_{N}+A_{N} N_{R} A_{\rho} \gamma_{R}$ the adjoint equation becomes just the wave equation (second order in time), rather then the system of two equations of first order.

\section{Sensitivity of optimal controls in $U=L_{2}(\Gamma \times(0, T))$}

By using variational definition of the projector operator $P_{U_{a d}}$ we infer the following characterization of the optimal control.

$$
\left(v_{\rho}^{0}+C_{\rho} v_{\rho}^{0}+F_{\rho}, u-v_{\rho}^{0}\right)_{\Sigma} \geq 0 \quad \forall u \in U_{a d} .
$$

The above characterization leads to the following error inequality satisfied by the difference of two optimal controls corresponding to $\rho>0$ and $\rho=0$ and denoted respectively by $v_{\rho}$ and $v^{0}$.

$$
\begin{aligned}
& \left|v^{0}-v_{\rho}^{0}\right|_{\Sigma}^{2}-\left(C_{\rho}\left(v^{0}-v_{\rho}^{0}\right), v^{0}-v_{\rho}^{0}\right)_{\Sigma} \\
& \left.\quad \leq\left(\left(C_{0}-C_{\rho}\right) v^{0}, v^{0}-v_{\rho}^{0}\right)_{\Sigma}+\left(F_{0}-F_{\rho}\right), v^{0}-v_{\rho}^{0}\right)_{\Sigma} .
\end{aligned}
$$

Since $-C_{\rho}$ is nonnegative, we obtain:

$$
\left.\left|v^{0}-v_{\rho}^{0}\right|_{\Sigma}^{2} \leq\left(\left(C_{0}-C_{\rho}\right) v^{0}, v^{0}-v_{\rho}^{0}\right)_{\Sigma}+\left(F_{0}-F_{\rho}\right), v^{0}-v_{\rho}^{0}\right)_{\Sigma} .
$$

Therefore, sensitivity analysis is reduced to sensitivity analysis of operators $C_{\rho}$ and $F_{\rho}$ respectively.

The first step toward sensitivity analysis of optimal control is sensitivity analysis of state operator due to specified control input.

We define the control-state operator $L_{\rho} ; L_{2}(\Sigma) \mapsto C(H)$ as:

$$
L_{\rho} u \equiv\left[y_{\rho}, \frac{d}{d t} y_{\rho}\right]=Y_{\rho},
$$

where $y_{\rho}$ satisfies (11) with $f=0, y(0)=0, y_{t}(0)=0$. 
We recall trace operator denoted by $\left.\gamma y \equiv y\right|_{\Gamma}$. From Theorem 3 we have

$$
\begin{aligned}
L_{\rho} & \in \mathcal{L}\left(L_{2}(\Sigma) ; C(H)\right) \\
\gamma P_{1} L_{\rho} & \in \mathcal{L}\left(L_{2}(\Sigma) \mapsto H^{1}(\Sigma)\right), \\
\gamma_{R} P_{1} L_{\rho} & \in \mathcal{L}\left(L_{2}(\Sigma) \mapsto H^{1}\left(\Sigma_{R}\right)\right)
\end{aligned}
$$

We shall also introduce the following notation:

$$
K(v) \equiv\left[z, z_{t}\right]=Z \text {, where } z \text { satisfies }
$$

$$
\begin{aligned}
z_{t t} & =\Delta z \\
\frac{\partial}{\partial v} z+\varepsilon z_{t} & =0 \quad \text { on } \Sigma \\
\frac{\partial}{\partial v} z+\varepsilon z_{t}+A_{0}(z) & =v \quad \text { on } \Sigma_{R} \\
z(0)=z_{t}(0) & =0 \\
(K(v))(t) & =\int_{0}^{t} S_{0, t-s}\left(\begin{array}{c}
0 \\
A_{N} N_{R} v(s)
\end{array}\right) d s .
\end{aligned}
$$

We already know from Theorem 3

$$
\begin{aligned}
& K: L_{2}\left(\Sigma_{R}\right) \mapsto C(H), \quad \text { is bounded, } \\
& \left.P_{1} K v\right|_{\partial \Omega_{R}} \in H^{1}\left(\Sigma \cup \Sigma_{R}\right) \quad \forall v \in L_{2}\left(\Sigma_{R}\right) .
\end{aligned}
$$

Lemma 3 Let $u \in L_{2}(\Sigma)$. Then

$$
L_{\rho} u-L_{0} u=\rho^{2} L^{\prime} u+r_{1}(\rho),
$$

where

$$
L^{\prime} u=K\left(B\left(\gamma_{R} y_{0}\right)\right)=K\left(B\left(\gamma_{R} P_{1} L_{0} u\right)\right) \in C(H), \quad L^{\prime} \in \mathcal{L}\left(L_{2}(\Sigma) \mapsto C(H)\right)
$$

and

$$
\frac{\left|r_{1}(\rho)\right|_{C(H)}}{\rho^{2}} \mapsto 0 \quad \forall u \in L_{2}(\Sigma)
$$

Proof Denote $\hat{Y} \equiv L_{\rho} u-L_{0} u$. Then $\hat{Y}=\left[\hat{y}, \hat{y}_{t}\right]$ satisfies

$$
\begin{aligned}
\hat{y}_{t t} & =\Delta \hat{y} \quad \text { in } Q_{R}, \\
\frac{\partial}{\partial v} \hat{y}+\varepsilon \hat{y}_{t} & =0 \quad \text { on } \Sigma, \\
\frac{\partial}{\partial v} \hat{y}+\varepsilon \hat{y}_{t}+A_{0}(\hat{y})+B\left(\gamma_{R} y_{0}\right) \rho^{2} & =-B\left(\gamma_{R} \hat{y}\right) \rho^{2}+O_{\rho^{4}}\left(\gamma_{R} P_{1} L_{\rho} u\right) \quad \text { on } \Sigma_{R}, \\
\hat{y}(0)=\hat{y}_{t}(0) & =0,
\end{aligned}
$$


where for $u \in L_{2}(\Sigma)$ we have

$$
\frac{\left|O_{\rho^{4}}\left(\gamma_{R} y_{\rho}\right)\right|_{L_{2}\left(\Sigma_{R}\right)}}{\rho^{4}} \leq c\left|\gamma_{R} y_{\rho}(t)\right|_{L_{2}\left(H^{1}\left(\Gamma_{R}\right)\right)} \leq C|u|_{L_{2}(\Sigma)} .
$$

This last conclusion follows from the fact that for $Y_{\rho} \in C(H)$ we have by Theorem 3 $\gamma_{R} y_{\rho} \in L_{2}\left(H^{1}\left(\Gamma_{R}\right)\right)$ and the higher order term $\mathcal{O}\left(\rho^{4}\right)$ satisfies

$$
\left|\mathcal{O}\left(\rho^{4}\right)(z)\right|_{L_{2}(\Gamma)} \leq \rho^{4}|z|_{H^{1}(\Gamma)} .
$$

From (42) and regularity Theorem 3 we obtain for all $u \in L_{2}(\Sigma)$

$$
L^{\prime} u=-K\left(B\left(\gamma_{R} y_{0}\right)\right)=-K\left(B\left(\gamma_{R} P_{1} L_{0} u\right)\right) \in C(H),
$$

with $P_{1}: \mathbb{R}^{2} \mapsto \mathbb{R}^{1}, P^{1}(x, y) \equiv x$, so that $P_{1}^{T} u=(0, u)$. From (42)

$$
\hat{Y} \rho^{-2}-L^{\prime} u=K\left(B\left(\gamma_{R} \hat{y}\right)\right)+K\left(\rho^{-2} O_{\rho^{4}}\left(\gamma_{R} P_{1} L_{\rho} u\right)\right) .
$$

Using the fact that the operator $B$ is bounded from $H^{1 / 2}(\Gamma) \mapsto L_{2}(\Gamma)$ and $K$ : $L_{2}(\Sigma) \mapsto C(H)$ is bounded we obtain the estimate

$$
\begin{aligned}
\left|\frac{\hat{Y}}{\rho^{2}}-L^{\prime} u\right|_{C(H)} & \leq \rho^{2}\left|\frac{\hat{Y}}{\rho^{2}}\right|_{C(H)}+\rho^{-2}\left|O_{\rho^{4}}\left(\gamma_{R} P_{1} L_{\rho} u\right)\right|_{L_{2}(\Sigma)} \\
& \leq \rho^{2}\left|\frac{\hat{Y}}{\rho^{2}}\right|_{C(H)}+\rho^{2}|u|_{L_{2}(\Sigma)}
\end{aligned}
$$

where in the last step we have used regularity theorems and the bound in (43). Thus, taking $\rho$ sufficiently small gives first via (44)

$$
\left|\frac{\hat{Y}}{\rho^{2}}\right|_{C(H)} \leq C, \quad \forall u \in L_{2}(\Sigma) .
$$

Hence

$$
\left|B\left(\gamma_{R} \hat{y}\right)\right|_{C\left(L_{2}(\Gamma)\right.} \leq C|\hat{y}|_{C\left(H^{1}\left(\Omega_{R}\right)\right)} \leq C|\hat{Y}|_{C(H)}
$$

and

$$
\left|B\left(\gamma_{R} \hat{y}\right)\right|_{L_{2}(\Sigma)} \mapsto 0, \quad \text { when } \rho \rightarrow 0,
$$

consequently by (41)

$$
\mid K\left(\left.B\left(\gamma_{R} \tilde{y}\right)\right|_{C(H)} \rightarrow 0, \quad \text { as } \rho \rightarrow 0 .\right.
$$

This leads to

$$
r_{1}(\rho) \equiv K\left(B\left(\gamma_{R} \hat{y}\right) \rho^{2}+K\left(O_{\rho^{4}}\left(L_{\rho}(u)\right)\right),\right.
$$

where after recalling $\left.L_{\rho} u\right|_{\Gamma} \in L_{2}\left(H^{1}(\Gamma)\right.$ (hidden tangential-space regularity in (38)) we obtain 


$$
\left|r_{1}(\rho)\right|_{C(H)} \rho^{-2} \rightarrow 0,
$$

when $\rho \rightarrow 0$ for all $u \in U_{a d}$, as desired.

PDE interpretation of the derivative $\left(L_{T}\right)^{\prime}$ is given below.

$L_{T}^{\prime} u=Z(T)=\left[z(t), z_{t}(t)\right](t=T)$ where $z(t)$ satisfies:

$$
\begin{aligned}
z_{t t} & =\Delta z \quad \text { in } Q_{R}, \\
\frac{\partial}{\partial v} z+\varepsilon z_{t} & =0 \quad \text { on } \Sigma, \\
\frac{\partial}{\partial v} z+\varepsilon z_{t}+A_{0} z & =B \gamma_{R} P_{1} L_{0} u \quad \text { on } \Sigma_{R}, \\
Z(0) & =0 \quad \text { in } \Omega_{R} .
\end{aligned}
$$

The analysis of the adjoint operator follows from duality. We recall that the duality is always considered with respect to the norm in $H$ topologized by $\tilde{A}_{N}^{1 / 2}$ for the first coordinate. We recall that this norm accounts for the effect of Steklov's operator.

Lemma 4 Let $W \in H$. Then

$$
L_{\rho, T}^{*} W-L_{0, T}^{*} W=\rho^{2}\left(L_{T}^{*}\right)^{\prime} W+r_{2}(\rho),
$$

where

$$
\left[L_{T}^{*}\right]^{\prime}=\left(L_{T}^{\prime}\right)^{*} \in \mathcal{L}\left(H \mapsto L_{2}(\Sigma)\right)
$$

and

$$
\frac{\left|r_{2}(\rho)\right|_{L_{2}(\Sigma)}}{\rho^{2}} \rightarrow 0 \quad \forall W \in H
$$

Proof The proof follows by duality. Let $W \in H$ and $u \in L_{2}(\Sigma)$. By Lemma 3

$$
\begin{aligned}
& \left(L_{\rho, T}^{*} W-L_{0, T}^{*} W, u\right)_{\Sigma} \\
& \quad=\left(W, L_{\rho, T} u-L_{0, T} u\right)_{H}=\rho^{2}\left(W, L_{T}^{\prime} u\right)_{H}+\left(W, r_{1}(\rho)(T)\right)_{H} \\
& \quad=\rho^{2}\left(\left(L_{T}^{\prime}\right)^{*} W, u\right)_{\Sigma}+\left(r_{2}(\rho), u\right)_{\Sigma}
\end{aligned}
$$

where $\left(r_{2}(\rho), u\right)_{\Sigma}=\left(W, r_{1}(\rho)(T)\right)_{H} \leq|W|_{H}\left|r_{1}(\rho)(T)\right|_{H}$. Thus

$$
\left[L_{T}^{*}\right]^{\prime}=\left(L_{T}^{\prime}\right)^{*} \in \mathcal{L}\left(H \mapsto L_{2}(\Sigma)\right),
$$

and by using the structure of $r_{1}(\rho)(T)$

$$
r_{2}(\rho) \equiv\left[K _ { T } \left(B\left(\gamma_{R} P_{1}\left(\left[L_{\rho}-L_{0}\right]\right)\right]^{*} W+\left[K_{T}\left(O_{\rho^{4}}\left(\gamma_{R} P_{1} L_{\rho}\right)\right]^{*} W,\right.\right.\right.
$$

which, by duality and Lemma 3, exhibits the prescribed rate of convergence. 
We recall projector operators $P_{i}: \mathbb{R}^{2} \mapsto \mathbb{R}^{1}, i=1,2$ given by

$$
P_{1}(u, v)=u, \quad P_{2}(u . v)=v .
$$

Considering $P_{1}: H \mapsto L_{2}\left(\Omega_{R}\right)$ we introduce the adjoint $P_{1}^{*}: L_{2}(\Omega) \mapsto H$ given by

$$
P_{1}^{*}=P_{1}^{T} \tilde{A}_{N}^{-1},
$$

where

$$
P_{1}^{T} \phi=(\phi, 0), \quad P_{2}^{T} \phi=(0, \phi) .
$$

Note that with the above notation: $L_{T}^{\prime}(u)=K_{T}\left(B \gamma_{R} P_{1} L_{0} u\right)$ and recalling $\gamma_{R}^{*}=$ $A_{N} N_{R}$ we obtain

$$
\left[K_{T}\left(B\left(\gamma_{R} P_{1} L_{0}\right)\right]^{*}=L_{0}^{*} P_{1}^{*} A_{N} N_{R} B^{*} K_{T}^{*}\right.
$$

Since $K_{T}(v)=(K v)(T)$

$$
K_{T}(v)=\int_{0}^{T} S_{0, T-t}\left(\begin{array}{c}
0 \\
A_{N} N_{R} v(t)
\end{array}\right) d t
$$

we have

$$
\left[K_{T}\right]^{*} W(t)=\gamma_{R} P_{2} S_{0, T-t}^{*} W
$$

and by (47)

$$
\left(L_{T}^{\prime}\right)^{*} W=L_{0}^{*} P_{1}^{T} \tilde{A}_{N}^{-1} A_{N} N_{R} B^{*} \gamma_{R} P_{2} S_{0, T-}^{*} . W .
$$

The above can be interpreted as follows:

Let $\Psi(t) \equiv S_{0, T-t}^{*} W=\left[\psi_{1}(t), \psi_{2}(t)\right]$. Then $\gamma_{R} P_{2} S_{0, T-t}^{*} W=\gamma_{R} \psi_{2}(t)$.

Since $\left(L_{0}^{*} F\right)(t)=N^{*} A_{N} P_{2} \int_{t}^{T} S_{0, s-t}^{*} F(s) d s$ we obtain the following lemma:

\section{Lemma 5}

$$
\begin{aligned}
v(t) & \equiv\left(L_{T}^{\prime}\right)^{*} W(t)=\left(L_{0}^{*} P_{1}^{T} \tilde{A}_{N}^{-1} A_{N} N B^{*} \gamma_{R} \psi_{t}(\cdot)\right)(t) \\
& =\gamma P_{2} \int_{t}^{T} S_{0, s-t}^{*}\left(\begin{array}{c}
\tilde{A}_{N}^{-1} A_{N} N B^{*} \gamma_{R} \psi_{t}(s) \\
0
\end{array}\right) d s
\end{aligned}
$$

has (after some calculations) the following representation

$$
v(t)=\gamma \phi(t)
$$

where

$$
\begin{aligned}
\phi_{t t} & =\Delta \phi \\
\frac{\partial}{\partial \nu} \phi-\varepsilon \phi_{t} & =0 \quad \text { on } \Gamma,
\end{aligned}
$$




$$
\begin{aligned}
\frac{\partial}{\partial v} \phi-\varepsilon \phi_{t}+A_{0} \phi & =B^{*} \gamma_{R} \psi_{t} \quad \text { on } \Gamma_{R}, \\
\phi(T)=\phi_{t}(T) & =0 \\
\psi_{t t} & =\Delta \psi, \\
\frac{\partial}{\partial v} \psi-\varepsilon \psi_{t} & =0 \quad \text { on } \Gamma \\
\frac{\partial}{\partial v} \psi-\varepsilon \psi_{t}+A_{0} \psi & =0 \quad \text { on } \Gamma_{R}, \\
\Psi(T) & =W
\end{aligned}
$$

and $\Psi$ satisfies the following energy inequality

$$
|\Psi(t)|_{H}^{2}+\varepsilon \int_{0}^{t}\left|\psi_{t}(s)\right|_{\Gamma \cup \Gamma_{R}}^{2}+\varepsilon|\psi|_{H^{1}\left(\Gamma \cup \Gamma_{R}\right)}^{2} d s \leq C|W|_{H}^{2} .
$$

We note that the regularity of the system above is not obvious, when considering PDE representation (since $\left(B^{*} \gamma_{R} \psi_{t} \in L_{2}\left(0, T ; H^{-1 / 2}\left(\Gamma_{R}\right)\right)\right)$. However, this result can be easily deduced from semigroup argument applied to integral representation of $v(t)$ (as given in the Lemma) along with hidden regularity given in Theorem 3.

The above results lead to the sensitivity analysis of operators $C_{\rho}$ and $F_{\rho}$ introduced in (28)

Lemma 6 Let $u \in L_{2}(\Sigma), Y_{0}, Y_{D} \in H$. The following expansion holds

$$
\begin{aligned}
C_{\rho} u-C_{0} u & =\rho^{2} C^{\prime} u+o\left(\rho^{2}\right), \\
C^{\prime} & =\frac{1}{\alpha} L_{T}^{*} \mathcal{R}^{*} \mathcal{R} L_{T}^{\prime}+\frac{1}{\alpha}\left(L_{T}^{*}\right)^{\prime} \mathcal{R}^{*} \mathcal{R} L_{T} \in \mathcal{L}\left(L_{2}(\Sigma)\right), \\
F_{\rho}-F_{0} & =F^{\prime} \rho^{2}+o\left(\rho^{2}\right), \\
F^{\prime} & =\frac{1}{\alpha} L_{T}^{*} \mathcal{R}^{*} \mathcal{R} S_{0, T}^{\prime} Y_{0}+\frac{1}{\alpha}\left(L_{T}^{*}\right)^{\prime} \mathcal{R}^{*} \mathcal{R}\left[S_{0, T} Y_{0}-Y_{D}\right] \in L_{2}(\Sigma) .
\end{aligned}
$$

We establish the directional differentiability of the optimal controls with respect to the parameter $\rho=0^{+}$.

Theorem 6 We have the following expansion of the optimal control in $L_{2}(\Gamma \times$ $(0, T))$, with respect to the small parameter,

$$
v_{\rho}^{0}=v^{0}+\rho^{2} q+o\left(\rho^{2}\right)
$$

for $\rho>0$.

Proof From (36) and formulas in Lemma 6 we obtain

$$
\left|v_{\rho}^{0}-v^{0}\right|_{\Sigma} \leq\left|\left(C_{0}-C_{\rho}\right) v^{0}\right|_{\Sigma}+\left|F_{0}-F_{\rho}\right|_{\Sigma} \leq C \rho^{2}
$$


for $Y(0) \in H$. Therefore, there exists $q \in L_{2}(\Sigma)$ such that

$$
v_{\rho}=v_{0}+\rho^{2} q+o\left(\rho^{2}\right) .
$$

In order to find the representation for the Gateau differential, we will be using representation of optimal controls $v_{\rho}^{0}$ given in (27). We write

$$
\begin{aligned}
v_{\rho}^{0}-v^{0} & =P_{U_{a d}}\left[-C_{\rho} v_{\rho}^{0}+F_{\rho}\right]-P_{U_{a d}}\left[-C_{0} v^{0}+F_{0}\right] \\
& =P_{U_{a d}}^{\prime}\left(-C_{0} v^{0}+F_{0}\right)\left[\left(-C_{\rho}+C_{0}\right) v^{0}-C_{\rho}\left(v_{\rho}^{0}-v^{0}\right)+F_{\rho}-F_{0}\right]+o\left(\rho^{2}\right) \\
& =\left[P_{U_{a d}}\right]^{\prime}\left(-C_{0} v^{0}+F_{0}\right)\left[\left(-C_{0} q-C_{0}^{\prime} v^{0}+F^{\prime}\right]+o\left(\rho^{2}\right),\right.
\end{aligned}
$$

where we have been using the fact that $P_{a d}$ is Lipschitz on $L_{2}(\Sigma)$. Comparing leading terms in (52) and the last equality we obtain

$$
q=\left[P_{U_{a d}}\right]^{\prime}\left(-C_{0} v^{0}+F_{0}\right)\left[-C_{0} q-C_{0}^{\prime} v^{0}+F^{\prime}\right]
$$

Moreover, we assume that $\rho$ is a sufficiently small. By exploiting explicit representations of $C_{\rho}$ operators the function $q$ can be written as

$$
q=\left[P_{U_{a d}}\right]^{\prime}\left(-C_{0} v^{0}+F_{0}\right)\left[-L_{T}^{*} \mathcal{R}^{*} \mathcal{R} W(T)-\left(L_{T}^{*}\right)^{\prime} \mathcal{R}^{*} \mathcal{R}\left(Y^{0}(T)-Y_{D}\right)\right],
$$

where $Y^{0}(t)$ is the optimal trajectory and $W(t)=\left[w(t), w_{t}(t)\right]$ satisfies the state equation

$$
\begin{array}{ll}
\frac{\partial^{2} w}{\partial t^{2}}-\Delta w=0 & \text { in } \Omega_{R} \times(0, T), \\
\frac{\partial w}{\partial \eta}+\varepsilon w_{t}=q & \text { on } \Gamma \times(0, T), \\
\frac{\partial w}{\partial \eta}+\varepsilon w_{t}+A_{0}(w)+B\left(\gamma_{R} y^{0}\right)=0 & \text { on } \Gamma_{R} \times(0, T), \\
w(x, 0)=0 & \text { in } \Omega_{R}, \\
\frac{\partial w}{\partial t}(x, 0)=0 & \text { in } \Omega_{R} .
\end{array}
$$

By using Regularity Theorem 3 one easily obtains:

Lemma 7 Solution $w$ satisfies:

$$
|w(t)|_{1, \Omega_{R}}^{2}+\left|w_{t}\right|_{0, \Omega_{R}}^{2}+\int_{0}^{t}\left|w_{t}\right|_{0, \Gamma}^{2} \leq \frac{c}{\varepsilon} \int_{0}^{t}\left[\left|y^{0}\right|_{1 / 2, \Gamma_{R}}^{2}+|q|_{\Gamma}^{2}\right] .
$$

We shall also introduce the performance functional

$$
I(u)=\frac{1}{2}|\mathcal{R} W(T, x)|_{H}^{2} d x+\frac{\alpha}{2} \int_{0}^{T} \int_{\Gamma}|u|^{2} d x d t,
$$


and the adjoint equation

$$
\left.\begin{array}{lc}
\frac{\partial^{2} z}{\partial t^{2}}+\Delta z=0 & \text { in } \Omega_{R} \times(0, T), \\
\frac{\partial z}{\partial \eta}-\varepsilon z_{t}=0 & \text { on } \Gamma \times(0, T), \\
\frac{\partial z}{\partial \eta}-\varepsilon z_{t}+A_{0}(z)+B^{*}\left(\gamma_{R} p^{0}\right)=0 & \text { on } \Gamma_{R} \times(0, T), \\
Z(T)=W(T) & \text { in } \Omega_{R}
\end{array}\right\}
$$

and $P^{0}(t)=\Psi(t)$ satisfies the adjoint equation in Lemma 5 with $\Psi(T) \equiv$ $\mathcal{R}^{*} \mathcal{R}\left(Y^{0}(T)-Y_{d}\right)$ (see Remark 4$)$.

Lemma 8 Regularity for $z$.

$$
\begin{aligned}
& |z(t)|_{1, \Omega_{R}}^{2}+\left|z_{t}(t)\right|_{0, \Omega_{R}}^{2}+\varepsilon \int_{0}^{T}\left|z_{t}\right|_{\Gamma \cup \Gamma_{R}}^{2} d s \\
& \quad \leq|w(T)|_{1, \Omega_{R}}^{2}+\left|w_{t}(T)\right|_{0, \Omega_{R}}^{2}+\frac{c}{\varepsilon} \int_{0}^{T}\left|p^{0}\right|_{1 / 2, \Gamma_{R}}^{2}
\end{aligned}
$$

With the above notation, the formula (54) can be written as

$$
q=\left[P_{U_{a d}}\right]^{\prime}\left(-C_{0} v_{0}+F_{0}\right)\left[-\gamma z_{t}\right] .
$$

Then, the optimal control $q$ is characterized by

$$
\begin{aligned}
& \left(\mathcal{R} W(x, T ; q), \mathcal{R}(W(x, T ; u)-W(x, T ; q))+\int_{0}^{T} \int_{\Gamma} q(u-q) d x d t \geq 0\right. \\
& \quad \forall u \in S_{a d},
\end{aligned}
$$

where: $S_{a d}$ is a set of admissible controls such that

$$
\begin{aligned}
& S_{a d}=\left\{u \in L_{2}(\Gamma \times(0, T)) \mid u(x, t) \geq 0 \text { on the set } E_{0}=\left\{(x, t) \mid v^{0}(x, t)=0\right\},\right. \\
& u(x, t)<0 \text { on the set } E_{1}=\left\{(x, t) \mid v^{0}(x, t)=1\right\}, \\
& \left.\int_{0}^{T} \int_{\Gamma}\left(p_{t}^{0}+\alpha v^{0}\right) u d x d t=0\right\},
\end{aligned}
$$

where: $p_{t}^{0}$ is a adjoint state for $\rho=0, v^{0}$ is a optimal solution for $\rho=0$ such that $0 \leq v^{0}(x, t) \leq 1$.

We simplify (60) using the adjoint equation (58). After transformations we obtain the following maximum condition

$$
\int_{0}^{T} \int_{\Gamma}\left(z_{t}+\alpha q\right)(u-q) d x d t \geq 0 \quad \forall u \in S_{a d} .
$$


Theorem 7 For the hyperbolic problem

$$
\left.\begin{array}{ll}
\frac{\partial^{2} w}{\partial t^{2}}-\Delta w=0 & \text { in } \Omega_{R} \times(0, T), \\
\frac{\partial w}{\partial \eta}+\varepsilon w_{t}=u & \text { on } \Gamma \times(0, T), \\
\frac{\partial w}{\partial \eta}+\varepsilon w_{t}+A_{0}(w)+B\left(y^{0}\right)=0 & \text { on } \Gamma_{R} \times(0, T), \\
w(x, 0)=0 & \text { in } \Omega_{R}, \\
\frac{\partial w}{\partial t}(x, 0)=0 & \text { in } \Omega_{R},
\end{array}\right\}
$$

with the performance functional (57) with $w(T) \in L_{2}\left(\Omega_{R}\right)$ and $\alpha>0$, and with constraints on the control (61), there exists a unique optimal control $q$ which satisfies the maximum condition (62).

\section{Conclusions}

The approximation used in this paper is obtained from the asymptotic analysis of the energy functional for the stationary problem. The energy functional is written for the Laplacian. The approximation is governed by a small parameter which describes singular perturbations of the domain. Such perturbations can be considered as a defect in a real world. The results presented are obtained for a defect in the form of a circular hole. For applications in the structural mechanics, the Laplace operator is replaced by a system of linear elasticity, and the defects can be some cracks, cavities or some other singularities with geometrical boundaries. Our method applies for such situations as well. The difference encountered is that instead of a scalar wave equation one should consider dynamic system of elasticity. There are several works $[3,10,35$, 47] which furnish the same kind of approximation for the energy functional, with the explicit expressions for the first order term, which can be used in our framework. The only difficulty is that instead of scalar problem, vectorial system of elasticity should be considered. This is possible, since all the ingredients are in place, including the hidden regularity $[12,13,15]$.

In this paper the mixed initial-boundary value problems of hyperbolic type is considered. One could also consider similar optimal control problems defined for time delay hyperbolic systems. The ideas mentioned above will be developed in forthcoming papers.

Open Access This article is distributed under the terms of the Creative Commons Attribution Noncommercial License which permits any noncommercial use, distribution, and reproduction in any medium, provided the original author(s) and source are credited.

\section{Appendix: Asymptotic analysis with Steklov-Poincaré operator}

For the convenience of the reader we provide the asymptotic analysis used for the elliptic problem in a singularly perturbed geometrical domain. 
9.1 Steklov-Poincaré operator in the domain $C(R, \rho)=\mathbb{B}_{R} \backslash \overline{\mathbb{B}}_{\rho}$

The main result, we obtain is based on the expansion (66) of the Steklov-Poincare operator with respect to the parameter $\rho$. The expansion is established in Sect. 9.1 by an application of elementary Fourier analysis.

We consider the mapping $A_{\rho}: H^{1 / 2}\left(\Gamma_{R}\right) \mapsto H^{-1 / 2}\left(\Gamma_{R}\right)$ defined by the boundary value problem

$$
\begin{aligned}
-\Delta w_{\rho}=0 & \text { in } C(R, \rho), \\
w_{\rho}=v & \text { on } \Gamma_{R}=\partial \mathbb{B}_{R}, \\
\partial_{n} w_{\rho}=0 & \text { on } \Gamma_{\rho},
\end{aligned}
$$

and we set

$$
\partial_{n} w_{\rho}=A_{\rho}(v) \quad \text { on } \Gamma_{R} .
$$

By an elementary evaluation of the associated energy functional, we refer the reader to Sect. 9.2 for details, taking into account the relation which follows by integration by parts, we find that

$$
\left\langle A_{\rho}(v), v\right\rangle_{\Gamma_{R}}=\int_{C(R, \rho)}\left|\nabla w_{\rho}(v ; x)\right|^{2} d x
$$

and for $\rho>0, \rho$ small enough,

$$
\int_{C(R, \rho)}\left|\nabla w_{\rho}(v ; x)\right|^{2} d x=\int_{\mathbb{B}_{R}}\left|\nabla w_{0}(v ; x)\right|^{2} d x+\rho^{2} b\left(\Gamma_{R} ; v, v\right)+O\left(\rho^{4}\right),
$$

where $w_{0}$ denotes the solution in the intact domain without any hole, and the remainder $O\left(\rho^{4}\right)$ is uniformly bounded on bounded sets in the space $H^{1 / 2}\left(\Gamma_{R}\right)$.

By the properties of harmonic functions the second term can be represented in two spatial dimensions in the equivalent form of a line integral over the circle $\Gamma_{R}=\{x$ : $|x-\mathcal{O}|=R\}$ with the centre at the origin $\mathcal{O}$

$$
b\left(\Gamma_{R} ; u, u\right)=-\frac{1}{2 \pi R^{6}}\left[\left(\int_{\Gamma_{R}} u x_{1} d s\right)^{2}+\left(\int_{\Gamma_{R}} u x_{2} d s\right)^{2}\right] .
$$

Therefore, we obtain the expansion

$$
A_{\rho}=A_{0}+\rho^{2} B+O\left(\rho^{4}\right),
$$

in the operator norm $\mathcal{L}\left(H^{1 / 2}\left(\Gamma_{R}\right) ; H^{-1 / 2}\left(\Gamma_{R}\right)\right)$.

\subsection{Compactness of asymptotic energy expansion}

In this section we provide a simple proof for (66) which is equivalent $[42,46]$ to (64). 
Remark 8 We refer e.g., to $[42,46]$ for the derivation of topological derivatives of the energy functionals for a class of elliptic boundary value problems including the linear elasticity. However, in our applications the form of the first term of the energy expansion should have some specific properties, therefore, we use the equivalent form of the topological derivative of the energy, which is bounded in the Sobolev energy space. The same property is required in the topological sensitivity analysis of the contact problems $[46,47]$.

Let $0 \in \Omega$ and $\mathbb{B}_{R}$ be a ball around 0 , while $C(\rho, R)$ is a ring $C(\rho, R)=\{\mathbf{x} \mid \rho<$ $\|\mathbf{x}\|<R\}$ with inner boundary $\Gamma_{\rho}$ and outer boundary $\Gamma_{R}$. Additionally we use notation $\Omega_{R}=\Omega \backslash \overline{\mathbb{B}}_{R}$, We consider functions $u \in H^{1}\left(\Omega_{R}\right)$ with traces (still denoted by $u$ ) on $\Gamma_{R}$ belonging to $H^{1 / 2}\left(\Gamma_{R}\right)$. The following implication is true

$$
\|u\|_{H^{1}\left(\Omega_{R}\right)} \leq \Lambda_{0} \quad \Longrightarrow \quad\|u\|_{H^{1 / 2}\left(\Gamma_{R}\right)} \leq \Lambda(R),
$$

and since $R$ is fixed, we shall omit it, writing $\Lambda$ instead of $\Lambda(R)$ (by $\Lambda$ we shall denote generic constant depending only on $\left.\Lambda_{0}\right)$. Finally, we denote by $(r, \phi)$ spherical coordinates around 0 .

From the fact that $u \in H^{1 / 2}\left(\Gamma_{R}\right)$ follows the existence of the Fourier series expansion in terms of $\phi$ :

$$
u=\frac{1}{2} a_{0}+\sum_{k=1}^{\infty}\left(a_{k} \sin k \phi+b_{k} \cos k \phi\right)
$$

with coefficients satisfying

$$
\sum_{k=1}^{\infty} \sqrt{1+k^{2}}\left(a_{k}^{2}+b_{k}^{2}\right) \leq \Lambda
$$

This implies two important for us properties:

$$
\sum_{k=1}^{\infty}\left(a_{k}^{2}+b_{k}^{2}\right) \leq \Lambda, \quad \sum_{k=1}^{\infty} k\left(a_{k}^{2}+b_{k}^{2}\right) \leq \Lambda .
$$

Now we shall consider in $\mathbb{B}_{R}$ the solution of the Laplace equation with Dirichlet boundary condition on $\Gamma_{R}$ coinciding with $u$, denoted by $w$, and the solution of the same equation in $C(\rho, R)$, with the same condition on $\Gamma_{R}$ and homogeneous Neumann condition on $\Gamma_{\rho}$, denoted by $w_{\rho}$. We define energies

$$
E(u)=\int_{\mathbb{B}_{R}}\|\nabla w\|^{2} d S, \quad E_{\rho}(u)=\int_{C(\rho, R)}\left\|\nabla w_{\rho}\right\|^{2} d S
$$

which depend on $u$ via boundary conditions. Our goal is to prove that $E_{\rho}$ has an expansion in which the remainder is uniformly bounded. More precisely this can be expressed as follows. 
Lemma 9 The energy $E_{\rho}(u)$ admits the expansion, for $\rho>0, \rho>0$ small enough,

$$
E_{\rho}(u)=E(u)+\rho^{2} b\left(\Gamma_{R} ; u, u\right)+\mathcal{R}(u),
$$

where

$$
|\mathcal{R}(u)| \leq \Lambda \rho^{4}
$$

uniformly on any fixed compact set in $H^{1}\left(\Omega_{R}\right)$, i.e. $\Lambda$ depends on this set only.

Proof Since any compact set may be covered by finite number of balls, it is enough to prove the Lemma for a fixed ball in $H^{1}\left(\Omega_{R}\right)$. We may therefore assume that (67) holds. The proof will consist in obtaining explicit formulas for $w$ and $w_{\rho}$ as series, using the well known methods, similarly as in [42]. Then the energies may be computed exactly and the desired property of the remainder $\mathcal{R}(u)$ proven.

Constructing $w$ from the Fourier series of its boundary condition we get

$$
w=\frac{1}{2} a_{0}+\sum_{k=1}^{\infty}\left(\frac{r}{R}\right)^{k}\left(a_{k} \sin k \phi+b_{k} \cos k \phi\right) .
$$

Similarly, for $w_{\rho}$ in $C(\rho, R)$ holds

$$
w_{\rho}=\frac{1}{2} a_{0}+\sum_{k=1}^{\infty} v_{k}(\rho)\left(a_{k} \sin k \phi+b_{k} \cos k \phi\right),
$$

where

$$
v_{k}(\rho)=A_{k} r^{k}+B_{k} \frac{1}{r^{k}},
$$

and

$$
\begin{aligned}
A_{k} R^{k}+B_{k} \frac{1}{R^{k}} & =1, \\
k A_{k} \rho^{k-1}-B_{k} \frac{1}{\rho^{k+1}} & =0 .
\end{aligned}
$$

Hence

$$
A_{k}=\frac{R^{k}}{R^{2 k}+\rho^{2 k}}, \quad B_{k}=A_{k} \rho^{2 k}
$$

and finally

$$
v_{k}(\rho)=\frac{r^{k}}{R^{k}}+\frac{\rho^{2 k}}{R^{2 k}+\rho^{2 k}}\left(\frac{R^{k}}{r^{k}}-\frac{r^{k}}{R^{k}}\right) .
$$

Substituting this into the expansion for $w_{\rho}$ gives

$$
w_{\rho}=w+\sum_{k=1}^{\infty} \frac{\rho^{2 k}}{R^{2 k}+\rho^{2 k}}\left(\frac{R^{k}}{r^{k}}-\frac{r^{k}}{R^{k}}\right)\left(a_{k} \sin k \phi+b_{k} \cos k \phi\right):=w+z_{\rho}
$$


with

$$
z_{\rho}=\sum_{k=1}^{\infty} \frac{\rho^{2 k}}{R^{2 k}+\rho^{2 k}}\left(\frac{R^{k}}{r^{k}}-\frac{r^{k}}{R^{k}}\right)\left(a_{k} \sin k \phi+b_{k} \cos k \phi\right) .
$$

For any function $f$ we denote by $f_{/ r}, f_{/ \phi}$ the partial derivatives with respect to the polar coordinates, thus the norm of the gradient with respect to the cartesian coordinates takes the form

$$
\|\nabla f\|^{2}=f_{/ r}^{2}+\frac{1}{r^{2}} f_{/ \phi}^{2}
$$

and therefore

$$
\begin{aligned}
E_{\rho}(u)= & \int_{C(\rho, R)}\left\|\nabla w+\nabla z_{\rho}\right\|^{2} d S \\
= & E(u)+\int_{C(\rho, R)}\left[\left(z_{\rho} / r\right)^{2}+\frac{1}{r^{2}}\left(z_{\rho / \phi}\right)^{2}\right] d S \\
& +2 \int_{C(\rho, R)}\left[w_{/ r} z_{\rho / r}+\frac{1}{r^{2}} w_{/ \phi} z_{\rho / \phi}\right] d S \\
& -\int_{\mathbb{B}_{\rho}}\|\nabla w\|^{2} d S \\
:= & E(u)+I_{1}+I_{2}+I_{3} .
\end{aligned}
$$

Now we have

$$
\begin{aligned}
z_{\rho / r} & =-\sum_{k=1}^{\infty} \frac{\rho^{2 k}}{R^{2 k}+\rho^{2 k}} k \frac{1}{r}\left(\frac{R^{k}}{r^{k}}-\frac{r^{k}}{R^{k}}\right)\left(a_{k} \sin k \phi+b_{k} \cos k \phi\right), \\
\frac{1}{r} z_{\rho / \phi} & =\sum_{k=1}^{\infty} \frac{\rho^{2 k}}{R^{2 k}+\rho^{2 k}} k \frac{1}{r}\left(\frac{R^{k}}{r^{k}}-\frac{r^{k}}{R^{k}}\right)\left(a_{k} \cos k \phi-b_{k} \sin k \phi\right) .
\end{aligned}
$$

After taking into account the orthogonality of trigonometric functions on $[0,2 \pi]$ and integrating with respect to $\phi$ one gets

$$
I_{1}=\frac{1}{\pi} \sum_{k=1}^{\infty}\left(\frac{\rho^{2 k}}{R^{2 k}+\rho^{2 k}}\right)^{2} k^{2}\left(a_{k}^{2}+b_{k}^{2}\right) \cdot I_{\rho k},
$$

where

$$
I_{\rho k}=\int_{\rho}^{R}\left[\left(\frac{R^{k}}{r^{k+1}}+\frac{r^{k-1}}{R^{k}}\right)^{2}+\left(\frac{R^{k}}{r^{k+1}}-\frac{r^{k-1}}{R^{k}}\right)^{2}\right] r d r .
$$

Since, after integration

$$
I_{\rho k}=\frac{1}{k}\left[\frac{R^{2 k}}{\rho^{2 k}}-\frac{\rho^{2 k}}{R^{2 k}}\right]
$$


we obtain

$$
I_{1}=\frac{1}{\pi} \sum_{k=1}^{\infty}\left(\frac{\rho^{2 k}}{R^{2 k}+\rho^{2 k}}\right)^{2} k\left(a_{k}^{2}+b_{k}^{2}\right)\left[\frac{R^{2 k}}{\rho^{2 k}}-\frac{\rho^{2 k}}{R^{2 k}}\right] .
$$

In order to compute $I_{2}$ we observe that

$$
\begin{aligned}
& w_{/ r}=\sum_{k=1}^{\infty} k \frac{r^{k-1}}{R^{k}}\left(a_{k} \sin k \phi+b_{k} \cos k \phi\right), \\
& w_{/ \phi}=\sum_{k=1}^{\infty} k \frac{r^{k}}{R^{k}}\left(a_{k} \cos k \phi-b_{k} \sin k \phi\right)
\end{aligned}
$$

and after easy computations

$$
I_{2}=0
$$

There remains $I_{3}$. It has the form

$$
I_{3}=-\int_{\mathbb{B}_{\rho}}\|\nabla w\|^{2} d S=-\int_{\mathbb{B}_{\rho}}\left(w_{/ r}^{2}+\frac{1}{r^{2}} w_{/ \phi}^{2}\right) d S,
$$

and in view of the written above expressions for $w_{/ r}, w_{/ \phi}$ and orthogonality

$$
I_{3}=-\frac{1}{\pi} \sum_{k=1}^{\infty} k^{2}\left(a_{k}^{2}+b_{k}^{2}\right) \int_{0}^{\rho} \frac{r^{2 k-2}}{R^{2 k}} r d r .
$$

Finally

$$
I_{3}=-\frac{1}{2 \pi} \sum_{k=1}^{\infty} \frac{\rho^{2 k}}{R^{2 k}} k\left(a_{k}^{2}+b_{k}^{2}\right) .
$$

There remains to observe that, for $\rho \leq \frac{1}{2} R$,

$$
\frac{\rho^{2 k}}{R^{2 k}+\rho^{2 k}}=\frac{\rho^{2 k}}{R^{2 k}}\left[1-\frac{\rho^{2 k}}{R^{2 k}}+\left(\frac{\rho^{2 k}}{R^{2 k}}\right)^{2}+\ldots\right] .
$$

Collecting the formulas (73), (74), (75) we may single out the first terms containing $\rho^{2}$ and the rest, which in view of the regularity of boundary conditions and implied by this inequalities (67) is uniformly bounded by $\Lambda \rho^{4}$.

It is worth noticing that as a byproduct of this proof we have once again derived the formula for energy correction $b\left(\Gamma_{R} ; u, u\right)$.

\section{References}

1. Ammari, H.: An inverse initial boundary value problem for the wave equation in the presence of imperfections of small volume. SIAM J. Control Optim. 41, 1194-1211 (2002) 
2. Berezin, F.A., Faddeev, L.D.: Remark on the Schrödinger equation with singular potential. Dokl. Akad. Nauk SSSR 137, 1011-1014 (1961). (Engl transl. in Soviet Math. Dokl. 2, 372-375 (1961))

3. Cardone, G., Nazarov, S.A., Sokołowski, J.: Asymptotic analysis, polarization matrices and topological derivatives for piezoelectric materials with small voids. SIAM J. Control Optim. 48, 3925-3961 (2010)

4. Frémiot, G., Horn, W., Laurain, A., Rao, M., Sokołowski, J.: On the analysis of boundary value problems in nonsmooth domains. Dissertat. Math. 462 (2009)

5. Hendrickson, E., Lasiecka, I.: Numerical approximations and regularizations of Riccati equations arising in hyperbolic dynamics with unbounded control operators. Comput. Optim. Appl. 2, 343-390 (1993)

6. Hendrickson, E., Lasiecka, I.: Finite dimensional approximations of boundary control problems arising in partially observed hyperbolic systems. Dyn. Contin. Discrete Impuls. Syst. 1, 101-142 (1995)

7. Hormander, L.: The Analysis of Linear Partial Differential Operators, vol. III. Springer, Berlin (1985)

8. Jackowska, L., Sokołowski, J., Żochowski, A., Henrot, A.: On numerical solution of shape inverse problems. Comput. Optim. Appl. 23, 231-255 (2002)

9. Karpeshina, Yu.E., Pavlov, B.S.: Interaction of the zero radius for the biharmonic and the polyharmonic equation. Mat. Zametki 40, 49-59 (1986) (in Russian)

10. Khludnev, A., Novotny, A., Sokołowski, J., Żochowski, A.: Shape and topology sensitivity analysis for cracks in elastic bodies on boundaries of rigid inclusions. J. Mech. Phys. Solids 57, 1718-1732 (2009)

11. Kurasov, P., Posilicano, A.: Finite speed of propagation and local boundary conditions for wave equations with point interactions. Proc. Am. Math. Soc. 133, 3071-3078 (2005)

12. Lagnese, J.: Stabilization of Thin Plates. SIAM, Philadelphia (1989)

13. Lagnese, J., Leugering, G.: Domain Decomposition Methods in Optimal Control of Partial Differential Equations. Birkhäuser, Basel (2004)

14. Landkof, N.S.: Fundamentals of Modern Potential Theory. Nauka, Moscow (1966) (in Russian)

15. Lasiecka, I.: Control Theory for Coupled PDE's. CBMS-SIAM-NSF Lecture Notes. SIAM, Philadelphia (2002)

16. Lasiecka, I., Lions, J.L., Triggiani, R.: Non-homogeneous boundary value problems for second order hyperbolic operators. J. Math. Pures Appl. 65, 149-192 (1986)

17. Lasiecka, I., Sokołowski, J.: Sensitivity analysis of constrained optimal control problem for wave equation. SIAM J. Control Optim. 29, 1128-1149 (1991)

18. Lasiecka, I., Triggiani, R.: Sharp regularity results for second order hyperbolic equations of Neumann type. Ann Mat. Pura Appl. CLVII, 1128-1149 (1990)

19. Lasiecka, I., Triggiani, R.: Regularity theory of hyperbolic equations with non-homogeneous Neumann boundary conditions. J. Differ. Equ. 94, 112-164 (1991)

20. Lasiecka, I., Triggiani, R.: Control Theory for Partial Differential Equations. Cambridge University Press, Cambridge (2000)

21. Lasiecka, I., Triggiani, R., Zhang, X.: In: Differential Geometric Methods in the Control of Partial Differential Equations. Contemporary Math., pp. 227-327. AMS, Providence (2000)

22. Lions, J.L.: Optimal Control of Systems Governed by Partial Differential Equations. Springer, Berlin (1971)

23. Lions, J.L., Magenes, E.: Non-Homogeneous Boundary Value Problems and Applications, vols. 1 and 2. Springer, Berlin (1972)

24. Malanowski, K.: Stability and sensitivity analysis for optimal control problems with control-state constraints. Dissertat. Math. 394 (2001)

25. Malanowski, K., Sokołowski, J.: Sensitivity of solutions to convex, control constrained optimal control problems for distributed parameter systems. J. Math. Anal. Appl. 120, 240-263 (1986)

26. Maz'ya, V., Nazarov, S., Plamenevskij, B.: Asymptotic Theory of Elliptic Boundary Value Problems in Singularly Perturbed Domains, vol. 1. Birkhäuser, Basel (2000)

27. Nazarov, S.A.: Self-adjoint extensions of the Dirichlet problem operator in weighted function spaces. Mat. Sb. 137, 224-241 (1988) (English transl.: Math. USSR Sbornik 65, 229-247 (1990))

28. Nazarov, S.A.: Asymptotic conditions at a point, self-adjoint extensions of operators and the method of matched asymptotic expansions. Am. Math. Soc. Transl. 198, 77-125 (1999)

29. Nazarov, S.A., Slutskij, A.S., Sokołowski, J.: Topological derivative of the energy functional due to formation of a thin ligament on a spatial body. Acta Univ. Lodz., Folia Math. 12, 39-72 (2005)

30. Nazarov, S.A., Sokołowski, J.: Asymptotic analysis of shape functionals. J. Math. Pures Appl. 82, 125-196 (2003) 
31. Nazarov, S.A., Sokołowski, J.: Self-adjoint extensions of differential operators in application to shape optimization. C. R., Méc. 331, 667-672 (2003)

32. Nazarov, S.A., Sokołowski, J.: The topological derivative of the Dirichlet integral due to formation of a thin ligament. Sib. Math. J. 45, 341-355 (2004)

33. Nazarov, S.A., Sokołowski, J.: Self-adjoint extensions for elasticity system in application to shape optimization. Bull. Pol. Acad. Sci., Math. 52, 237-248 (2004)

34. Nazarov, S.A., Sokołowski, J.: Self-adjoint extensions for the Neumann Laplacian and applications. Acta Math. Sin. 22, 879-906 (2006)

35. Nazarov, S.A., Specovius-Neugebauer, M., Sokołowski, J.: Polarization matrices in anisotropic elasticity. Asymptot. Anal. 68, 189-221 (2010)

36. Pavlov, B.S.: The theory of extension and explicitly soluble models. Usp. Mat. Nauk 42, 99-131 (1987) (Engl. transl. Soviet Math. Surveys 42, 127-168 (1987))

37. Pólya, G., Szegö, G.: Isoperimetric Inequalities in Mathematical Physics. Princeton University Press, Princeton (1951)

38. Sakamoto, R.: Hyperbolic Boundary Value Problems. Cambridge University Press, Cambridge (1982)

39. Sokołowski, J.: Differential stability of solutions to constrained optimization problems. Appl. Math. Optim. 13, 97-115 (1985)

40. Sokołowski, J.: Sensitivity analysis of control constrained optimal control problems for distributed parameter systems. SIAM J. Control Optim. 25, 1542-1556 (1987)

41. Sokołowski, J.: Shape sensitivity analysis of boundary optimal control problems for parabolic systems. SIAM J. Control Optim. 26, 763-787 (1988)

42. Sokołowski, J., Żochowski, A.: On topological derivative in shape optimization. SIAM J. Control Optim. 37, 1251-1272 (1999)

43. Sokołowski, J., Żochowski, A.: Topological derivative for optimal control problems. Control Cybern. 28, 611-626 (1999)

44. Sokołowski, J., Żochowski, A.: Topological derivatives for elliptic problems. Inverse Probl. 15, 123134 (1999)

45. Sokołowski, J., Żochowski, A.: Optimality conditions for simultaneous topology and shape optimization. SIAM J. Control Optim. 42, 1198-1221 (2003)

46. Sokołowski, J., Żochowski, A.: Modelling of topological derivatives for contact problems. Numer. Math. 102, 145-179 (2005)

47. Sokołowski, J., Żochowski, A.: Asymptotic analysis and topological derivatives for shape and topology optimization of elasticity problems in two spatial dimensions. Eng. Anal. Bound. Elem. 32, 533544 (2008)

48. Sokołowski, J., Zolesio, J.-P.: Introduction to Shape Optimization. Shape Sensitivity Analysis. Springer, Berlin (1992) 